TRANSACTIONS OF THE

AMERICAN MATHEMATICAL SOCIETY

Volume 358, Number 5, Pages 2183-2203

S 0002-9947(05)03749-9

Article electronically published on August 1, 2005

\title{
COHOMOLOGY THEORIES BASED ON GORENSTEIN INJECTIVE MODULES
}

\author{
JAVAD ASADOLLAHI AND SHOKROLLAH SALARIAN
}

\begin{abstract}
In this paper we study relative and Tate cohomology of modules of finite Gorenstein injective dimension. Using these cohomology theories, we present variations of Grothendieck local cohomology modules, namely Gorenstein and Tate local cohomology modules. By applying a sort of AvramovMartsinkovsky exact sequence, we show that these two variations of local cohomology are tightly connected to the generalized local cohomology modules introduced by J. Herzog. We discuss some properties of these modules and give some results concerning their vanishing and non-vanishing.
\end{abstract}

\section{INTRODUCTION}

Let $R$ be a commutative Noetherian ring. The notion of the G-dimension of a finitely generated module over such a ring was introduced by Auslander and Bridger $\mathrm{AB}$. Over a Gorenstein ring the modules of G-dimension 0 are precisely the maximal Cohen-Macaulay modules. They play a central rôle in the study of the category of finite $R$-modules. Avramov and Martsinkovsky [AM] studied the so-called relative and Tate cohomology theories in the subcategory of modules of finite G-dimension and made an intensive study of the interaction between the three cohomology theories: i.e. the absolute, the relative and the Tate cohomology theories.

The subject of relative homological algebra goes back to S. Eilenberg and J.C. Moore in their 1965 AMS Memoir [EM] and was further studied by Mac Lane [Ma]. With this approach modules other than projectives are allowed in resolutions. And so in $\mathrm{AM}$ the modules of G-dimension 0 were used as the relative projectives.

Another cohomology theory, Tate cohomology, introduced through complete resolutions, has been the subject of several recent expositions, in particular by Buchweitz $[\mathrm{Bu}]$ and Cornick and Kropholler $\mathrm{CK}$.

The main result of $\mathrm{AM}$ ] shows that there is a very close connection between these theories. To give their main result, let $\operatorname{Ext}_{\mathcal{G}}^{n}(,$,$) denote the relative cohomology$ functor computed by treating the modules of G-dimension 0 as the projectives and let $\widehat{\operatorname{Ext}}_{R}^{n}($, ) denote the Tate cohomology functor. Then there exists an exact sequence of functors

$$
0 \rightarrow \operatorname{Ext}_{\mathcal{G}}^{1} \rightarrow \operatorname{Ext}_{R}^{1} \rightarrow \cdots \rightarrow \operatorname{Ext}_{\mathcal{G}}^{n} \rightarrow \operatorname{Ext}_{R}^{n} \rightarrow{\widehat{\operatorname{Ext}_{R}}}_{R}^{n} \rightarrow \operatorname{Ext}_{\mathcal{G}}^{n+1} \rightarrow \cdots
$$

Received by the editors April 23, 2003 and, in revised form, June 15, 2004.

2000 Mathematics Subject Classification. Primary 13D05, 13D45, 13H10, 13D03, 55N35.

Key words and phrases. Gorenstein injective coresolutions, local cohomology modules, Tate cohomology, Gorenstein rings, Gorenstein dimension.

This research was supported in part by a grant from IPM (No. 82130113 and No. 82130118). 
On the other hand G-dimension 0 modules are called Gorenstein projective by Enochs and collaborators [EJ1] and so recall their rôle in a relative cohomology theory. The way they defined G-projective (short for Gorenstein projective) modules can be dualized and allows one to define Gorenstein injective (G-injective for short) modules. These notions generalize the usual projective and injective modules.

In light of the above and motivated by the work in [AM] one can treat the Ginjective modules as injectives and get dually defined relative cohomology functors which will be denoted $\operatorname{ext}_{\mathcal{G I}}^{n}(, N)$. These functors have this homological property: their vanishing defines a numerical invariant (the G-injective dimension) which refines the classical injective dimension with equality holding between the dimensions when the injective dimension is finite.

Moreover, by using a method which is similar (or dual) to what is called the pitchfork construction (and originally used by Auslander and Buchweitz to construct maximal Cohen-Macaulay approximations of modules over commutative Gorenstein rings) we construct for any module $N$ of finite G-injective dimension the complete coresolution $N \rightarrow \mathbf{I} \rightarrow \mathbf{T}$. Using this, for each $R$-module $M$ and each $n \in \mathbb{Z}$, a Tate cohomology group is defined by the equality $\widehat{\operatorname{ext}}_{R}^{n}(M, N)=\mathrm{H}^{n} \operatorname{Hom}_{R}(M, \mathbf{T})$. We will show that the functors $\widehat{\operatorname{ext}}_{R}^{n}$ are independent of the choice of resolutions and liftings. Functoriality in both the co and contravariant variables will also be discussed. Moreover, we will give an exact sequence of functors

$$
0 \rightarrow \operatorname{ext}_{\mathcal{G I}}^{1} \rightarrow \operatorname{Ext}_{R}^{1} \rightarrow \cdots \rightarrow \operatorname{ext}_{\mathcal{G I}}^{n} \rightarrow \operatorname{Ext}_{R}^{n} \rightarrow \widehat{\operatorname{ext}}_{R}^{n} \rightarrow \operatorname{ext}_{\mathcal{G I}}^{n+1} \rightarrow \cdots
$$

that relates absolute cohomology and the relative and Tate cohomologies.

The heart of the paper is in section 5. Here we give a generalization of local cohomology theory of Grothendieck (see [Gr]) using the ext ${ }_{\mathcal{G}}^{i}$ functors. For this let $\overline{\mathcal{G I}}$ denote the full subcategory of the category of $R$-modules whose objects admit some $\mathcal{G} \mathcal{I}$-proper coresolution (see section 2 below for definitions). Let $\mathfrak{a}$ be an ideal of $R$. For any $R$-module $M$ and any $R$-module $N$ in $\overline{\mathcal{G I}}$, we shall define for all $i \in \mathbb{Z}$, the $R$-module $\underset{\overrightarrow{n \in \mathbb{N}}}{\lim } \operatorname{ext}_{\mathcal{G I}}^{i}\left(M / \mathfrak{a}^{n} M, N\right)$ to be the $i$ th Gorenstein local cohomology of $M$ and $N$ with respect to $\mathfrak{a}$. We denote these modules by $G H_{\mathfrak{a}}^{i}(M, N)$. With $M=R$ one obtains the local cohomology introduced by Grothendieck. We discuss some properties of these modules and give some results concerning their vanishing and non-vanishing.

The basic structure of the paper is as follows: after the Introduction we summarize some preliminaries and basic facts about complexes of modules, G-injective dimension and $\mathcal{G} \mathcal{I}$-proper coresolutions in section 2 . In section 3 we introduce and study briefly the relative (respectively Tate) cohomology on the $\overline{\mathcal{G I}}$ (respectively, modules of finite G-injective dimension, denoted by $\widetilde{\mathcal{G I}}$ ). We also examine the homological properties of the cohomology functors ext $\operatorname{GI}_{\mathcal{I}}$ and $\widehat{\operatorname{ext}}_{R}$. The basic problem here is the relation between functor ext $\mathcal{G I}_{\mathcal{I}}$ and functor $\operatorname{Ext}_{\mathcal{G}}$ of $[\mathrm{AM}]$. In section 4 we deal with the balance problem of the (bi)functor 'Hom'. Balance means that we have two specific kinds of (co)resolutions of each of two variables, the $\mathcal{G}$-proper resolution of $M$ and $\mathcal{G} \mathcal{I}$-proper coresolution of $N$, each of which can be used to compute the relative derived functors. We conclude, in particular, that 'Hom' is balanced over (arbitrary) commutative Noetherian ring, when G-dimension of $M$ and G-injective dimension of $N$ are both finite. Furthermore, we discuss the balance of the functors $\widehat{\operatorname{Ext}}_{R}^{n}$ and $\widehat{\operatorname{ext}}_{R}$. As a result we show that the $i$ th Auslander's $\delta$-invariant for a 
finitely generated $R$-module $M[\mathrm{Bu}$ ] can be computed over a Gorenstein local ring as the dimension of the $k$-vector space $\operatorname{Ker}\left(\operatorname{Ext}_{R}^{i}(M, k) \longrightarrow \widehat{\operatorname{ext}}_{\mathcal{G I}}^{i}(M, k)\right)$.

In section 5 we give a pleasant application of our results in the previous sections. Grothendieck-Hartshorne local cohomology theory has become indispensable for many mathematicians working in the theory of commutative Noetherian rings. There are several approaches to this cohomology: it can be computed as the right derived functors of the $\mathfrak{a}$-torsion functor $\Gamma_{\mathfrak{a}}(-)=\bigcup_{n \in \mathbb{N}} 0::_{(-)} \mathfrak{a}^{n}$ as well as using ' $\operatorname{Ext}_{R}$ ' functors, that is, the $i$ th local cohomology of $N$ with respect to $\mathfrak{a}$, denoted $H_{\mathfrak{a}}^{i}(N)$, is isomorphic to $\underset{\overrightarrow{n \in \mathbb{N}}}{\lim } \operatorname{Ext}_{R}^{i}\left(R / \mathfrak{a}^{n}, N\right)$. In view of this, for an arbitrary $R$-module $M$ and any $R$-module $N$ in $\overline{\mathcal{G I}}$, we consider the $R$-module $\lim \operatorname{ext}_{\mathcal{G} \mathcal{I}}^{i}\left(M / \mathfrak{a}^{n} M, N\right)$ as the $i$ th Gorenstein local cohomology module of $M$ and $N$ $\overrightarrow{n \in \mathbb{N}}$

with respect to $\mathfrak{a}$. The notation $G H_{\mathfrak{a}}^{i}(M, N)$ is used to indicate this. Correspondingly one can define for any $i \in \mathbb{Z}$ the $i$ th Tate cohomology of $M$ and $N$ with respect to $\mathfrak{a}$ as $\underset{n \in \mathbb{N}}{\operatorname{limext}} \widehat{C}_{R}^{i}\left(M / \mathfrak{a}^{n} M, N\right)$. We denote this by $\widehat{H}_{\mathfrak{a}}^{i}(M, N)$. In section 5 we exhibit, among other things, a close connection between these two local cohomology theories and generalized local cohomology of Herzog [He]. That is, we prove the existence of an exact sequence

$$
\begin{aligned}
0 & \rightarrow G H_{\mathfrak{a}}^{1}(M, N) \rightarrow H_{\mathfrak{a}}^{1}(M, N) \rightarrow \cdots \rightarrow G H_{\mathfrak{a}}^{n}(M, N) \rightarrow H_{\mathfrak{a}}^{n}(M, N) \\
& \rightarrow \widehat{H_{\mathfrak{a}}^{n}}(M, N) \rightarrow G H_{\mathfrak{a}}^{n+1}(M, N) \rightarrow \cdots .
\end{aligned}
$$

Section 6 is devoted to presenting some vanishing and non-vanishing results of Gorenstein local cohomology modules over Gorenstein rings. We show that Gorenstein local cohomology module $G H_{\mathfrak{a}}^{i}(M, N)$ vanishes for degrees more than G- $\operatorname{dim}_{R} M+\operatorname{dim} N$. Moreover we obtain a duality which is similar to Grothendieck Matlis duality.

Throughout the paper $R$ will denote a commutative Noetherian ring. All other notation is standard.

\section{Preliminaries}

We begin by reviewing some definitions and notation. Our complexes shall have differentials of degree -1 . A complex will be displayed as

$$
\mathbf{C}: \quad \cdots \longrightarrow C_{n+1} \stackrel{\partial_{n+1}^{\mathbf{C}}}{\longrightarrow} C_{n} \stackrel{\partial_{n}^{\mathbf{C}}}{\longrightarrow} C_{n-1} \longrightarrow \cdots .
$$

The $i$ th syzygy module of $\mathbf{C}$ is $\Omega^{i} \mathbf{C}=$ Coker $\partial_{\mathbf{i}+\mathbf{1}}^{\mathbf{C}}$. $\Sigma^{i} \mathbf{C}$ denotes the $i$ th shift of $\mathbf{C}$, that is, a complex with $n$th component equal to $\mathbf{C}_{n-i}$ and $\partial_{n}^{\Sigma^{i} \mathbf{C}}=(-1)^{i} \partial_{n-i}^{\mathbf{C}}$. The notation $\mathbf{C}_{>i}$ denotes the subcomplex of $\mathbf{C}$ with $n$th component equal to $\mathbf{C}_{n}$ for $n>i$ and to 0 for $n \leq i$.

If $\mathbf{B}$ and $\mathbf{C}$ are two complexes, a homomorphism $\beta: \mathbf{B} \rightarrow \mathbf{C}$ of degree $i$ is a sequence of $R$-linear maps $\beta_{n}: B_{n} \rightarrow C_{n+i}$ for all $n \in \mathbb{Z}$. A morphism of complexes is a chain map of degree 0 . By a quasi-isomorphism we mean a morphism $\beta: \mathbf{B} \rightarrow \mathbf{C}$ with $\mathrm{H}_{n}(\beta): \mathrm{H}_{n} \mathbf{B} \rightarrow \mathrm{H}_{n} \mathbf{C}$ bijective for all $n$.

A coresolution (of length $\leq l$ ) of a module $M$ is a quasi-isomorphism $\gamma: M \rightarrow \mathbf{E}$ with $E_{n}=0$ for all $n>0$ (and $E_{n}=0$ for all $n<-l$ ): it gives rise to an exact 
sequence

$$
\mathbf{E}^{+}: \quad 0 \longrightarrow M \longrightarrow E_{0} \longrightarrow E_{-1} \longrightarrow \cdots \longrightarrow E_{-n} \stackrel{\partial_{-n}}{\longrightarrow} E_{-n-1} \longrightarrow \cdots
$$

We sometimes use the convention $E_{-i}=E^{i}$ and so denote the coresolution $\mathbf{E}$ as

$$
\mathbf{E}^{+}: \quad 0 \rightarrow M \rightarrow E^{0} \rightarrow E^{1} \rightarrow \cdots E^{n} \stackrel{\partial^{n}}{\rightarrow} E^{n+1} \rightarrow \cdots
$$

Auslander and Bridger $\mathrm{AB}$ introduced the G-class $\mathrm{G}(R)$ as a generalization of the class of projective modules. In $\mathrm{AM}$ these modules are called totally reflexive modules. Because of their rôle in the relative cohomology theory it seems appropriate to call the modules in the G-class the Gorenstein projective modules. This is done by Enochs and collaborators; cf. e.g. EJ1. They also dualized the notion of a Gorenstein projective module and defined Gorenstein injective modules. So now let $\mathcal{I}=\mathcal{I}(R)$ denote the full subcategory of $\mathcal{C}(R)$ whose objects are injective $R$-modules, where $\mathcal{C}=\mathcal{C}(R)$ denotes the category of all $R$-module and $R$-homomorphisms.

Definition 2.1. Consider the complex $\mathbf{E}$ with $E_{n} \in \mathcal{I}$ such that

$$
\mathrm{H}_{n}(\mathbf{E})=\mathrm{H}_{n}\left(\operatorname{Hom}_{R}(I, \mathbf{E})\right)=0
$$

for all $n \in \mathbb{Z}$ and all $I \in \mathcal{I}$. The syzygies of this complex are called Gorenstein injective (G-injective for short) $R$-modules.

The class of all G-injective $R$-modules is denoted by $\mathcal{G I}$. Clearly $\mathcal{I} \subseteq \mathcal{G} \mathcal{I}$. Moreover by $[\mathrm{H} 2,2.6] \mathcal{G I}$ is injectively resolving. We recall that a class $\mathcal{X}$ of $R$ modules is called injectively resolving if it contains $\mathcal{I}$ and if for every short exact sequence $0 \rightarrow X^{\prime} \rightarrow X \rightarrow X^{\prime \prime} \rightarrow 0$ of $R$-modules with $X^{\prime} \in \mathcal{X}$, we can conclude that $X \in \mathcal{X}$ if and only if $X^{\prime \prime} \in \mathcal{X}$.

A coresolution $M \rightarrow \mathbf{E}$ is called a $\mathcal{G I}$-coresolution if $E_{n}$ belongs to $\mathcal{G I}$ for all $n \in \mathbb{Z}$. A $\mathcal{G I}$-coresolution $M \rightarrow \mathbf{E}$ is said to be strict if $E_{-n}$ is injective for all $n \geq 1$. Gorenstein injective dimension of an $R$-module $M, \operatorname{Gid}_{R} M$ is defined as

$$
\operatorname{Gid}_{R} M=\inf \left\{\begin{array}{c|c}
n \in \mathbb{N} \mid \begin{array}{c}
\text { there exists a } \mathcal{G} \mathcal{I} \text {-coresolution } \\
M \rightarrow \mathbf{E} \text { of length } \leq n
\end{array}
\end{array}\right\} .
$$

We use $\widetilde{\mathcal{I}}$ (respectively $\widetilde{\mathcal{G}} \mathcal{I}$ ) to denote the full subcategory of $\mathcal{C}$, whose objects are modules of finite injective (respectively G-injective) dimension. Trivially $\widetilde{\mathcal{I}} \subseteq \widetilde{\mathcal{G I}}$. A complex $\mathbf{C}$ in $\mathcal{C}$ is called $\mathcal{G} \mathcal{I}$-proper exact if the induced complex $\operatorname{Hom}_{R}(\mathbf{C}, G)$ is exact for all $G \in \mathcal{G} \mathcal{I}$. Since the injective cogenerator of $R$ belongs to $\mathcal{G} \mathcal{I}$, every $\mathcal{G I}$-proper exact complex is exact. A $\mathcal{G I}$-proper coresolution $M \rightarrow \mathbf{E}$ is a $\mathcal{G} \mathcal{I}$ coresolution such that its associated complex $\mathbf{E}^{+}$is $\mathcal{G I}$-proper exact. Note that by EJ2, 10.1.3], any injective coresolution of a module of finite injective dimension is $\mathcal{G I}$-proper exact. Let $\overline{\mathcal{G I}}$ denote the full subcategory of $\mathcal{C}$ whose objects are the modules admitting some $\mathcal{G} \mathcal{I}$-proper coresolution. It is clear that every split exact sequence is $\mathcal{G} \mathcal{I}$-proper. Moreover by [EJ2, 10.1.3], it is easy to see that every exact sequence $0 \rightarrow N \rightarrow N^{\prime} \rightarrow N^{\prime \prime} \rightarrow 0$ with id ${ }_{R} N^{\prime \prime}<\infty$ or $\operatorname{pd}_{R} N^{\prime \prime}<\infty$ is $\mathcal{G} \mathcal{I}$-proper. This implies that every strict $\mathcal{G I}$-coresolution $M \rightarrow \mathbf{E}$ of finite length is $\mathcal{G I}$-proper. So there is an inclusion of categories $\widetilde{\mathcal{G I}} \subseteq \overline{\mathcal{G I}}$. 


\section{Absolute, Relative and Tate cohomology of modules of finite GORENSTEIN INJECTIVE DIMENSION}

This section is motivated by Avramov and Martsinkovsky's elegant work [AM]. They studied absolute, relative and Tate cohomology of modules of finite Gorenstein dimension. In this section we deal with modules of finite G-injective dimension. Basically some of our results are proved using their techniques. So we omit some of the proofs and refer the reader to that source.

Let $N \in \overline{\mathcal{G I}}$ and consider a $\mathcal{G I}$-proper coresolution $N \rightarrow$ E. For every $n \in \mathbb{Z}$ and every $M \in \mathcal{C}$, define a relative cohomology group

$$
\operatorname{ext}_{\mathcal{G} \mathcal{I}}^{n}(M, N)=\mathrm{H}^{n}\left(\operatorname{Hom}_{R}(M, \mathbf{E})\right) \text {. }
$$

Now choose an injective coresolution $N \rightarrow \mathbf{I}$ and a morphism of complexes $\Psi: \mathbf{E} \rightarrow$ I that lifts the identity map of $N$ to obtain a homomorphism

$$
\partial_{\mathcal{G I}}^{n}(M, N): \operatorname{ext}_{\mathcal{G} \mathcal{I}}^{n}(M, N) \rightarrow \operatorname{Ext}_{R}^{n}(M, N),
$$

where

$$
\partial_{\mathcal{G} \mathcal{I}}^{n}(M, N)=\mathrm{H}^{n}\left(\operatorname{Hom}_{R}(M, \Psi)\right): \mathrm{H}^{n}\left(\operatorname{Hom}_{R}(M, \mathbf{E})\right) \rightarrow \mathrm{H}^{n}\left(\operatorname{Hom}_{R}(M, \mathbf{I})\right) .
$$

One can easily check that for $n \in \mathbb{Z}$,

$$
\operatorname{ext}_{\mathcal{G} \mathcal{I}}^{n}: \mathcal{C}(R) \times \overline{\mathcal{G I}}(R) \rightarrow \mathcal{C}(\mathbb{Z})
$$

is a functor and $\partial_{\mathcal{G} \mathcal{I}}^{n}$ is a morphism of functors $\operatorname{ext}_{\mathcal{G} \mathcal{I}}^{n} \rightarrow \operatorname{Ext}_{R}^{n}$. Moreover $\operatorname{ext}_{\mathcal{G} \mathcal{I}}^{n}$ and $\partial_{\mathcal{G} \mathcal{I}}^{n}$ are independent of the choice of coresolutions and liftings.

Remark 3.1. Let $R$ be a Gorenstein ring of finite dimension. Then by EJ2, 11.2.1] the class of G-injective modules is preenveloping, that is, every module admits a Ginjective preenvelope. So every $R$-module has a $\mathcal{G I}$-proper coresolution [EJ2, 8.1.3]. Using this, Enochs and Jenda EJ2, 12.1.4] have computed right derived functors of $\operatorname{Hom}_{R}(M, N)$. They denoted these right derived functors by $\operatorname{Gext}_{R}^{i}(M, N)$. As is clear, our definition of $\operatorname{ext}_{\mathcal{G}}^{i}(M, N)$ is identical with their definition provided $R$ is a finite-dimensional Gorenstein ring.

Our next two results can be proved by dualizing the proofs of similar (dual) results in [AM, Sec. 4], so we omit their proofs.

Theorem 3.2. Let $N \in \overline{\mathcal{G I}}$. Then

(1) $\partial_{\mathcal{G} \mathcal{I}}^{0}: \operatorname{ext}_{\mathcal{G I}}^{0} \rightarrow \operatorname{Hom}_{R}$ is an isomorphism and $\operatorname{ext}_{\mathcal{G I}}^{n}(, N)=0$ for all $n<0$.

(2) If $\operatorname{id}_{R} N<\infty$, then $\partial_{\mathcal{G} \mathcal{I}}^{n}(M, N)$ is bijective for all $n \in \mathbb{Z}$.

(3) For every $n \geq 0$, the following conditions are equivalent:

i) $\operatorname{Gid}_{R} N \leq n$.

ii) $\operatorname{ext}_{\mathcal{G I}}^{i}(, N)=0$ for all $i>n$.

iii) $\operatorname{ext}_{\mathcal{G I}}^{n+1}(, N)=0$.

iv) Each injective coresolution $N \rightarrow \mathbf{I}$ has $\Omega^{i} \mathbf{I} \in \mathcal{G I}$ for all $i \geq n$.

Theorem 3.3. (a) Let $N \in \overline{\mathcal{G I}}$ and $\mathbf{M}=0 \rightarrow M \rightarrow M^{\prime} \rightarrow M^{\prime \prime} \rightarrow 0$ be a $\mathcal{G} \mathcal{I}$-proper exact sequence of $R$-modules. Then there exists a long exact sequence of $\mathbb{Z}$-modules

$$
\begin{gathered}
\cdots \underset{\gamma_{\mathcal{I}}(\mathbf{M}, N)}{\longrightarrow} \operatorname{ext}_{\mathcal{G I}}^{n}\left(M^{\prime \prime}, N\right) \longrightarrow \operatorname{ext}_{\mathcal{G} \mathcal{I}}^{n}\left(M^{\prime}, N\right) \longrightarrow \operatorname{ext}_{\mathcal{G} \mathcal{I}}^{n}(M, N) \\
\operatorname{ext}_{\mathcal{G}}^{n+1}\left(M^{\prime \prime}, N\right) \longrightarrow \cdots .
\end{gathered}
$$


(b) Let $\mathbf{N}=0 \rightarrow N \rightarrow N^{\prime} \rightarrow N^{\prime \prime} \rightarrow 0$ be a $\mathcal{G}$-proper exact sequence of $R$-modules in $\overline{\mathcal{G I}}$. Then for any $R$-module $M$, there is a long exact sequence of $R$-modules

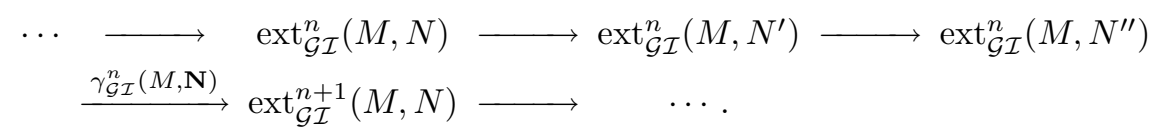

The connecting homomorphisms in both (a) and (b) are natural in $M$ and $N$ homomorphisms.

Proposition 3.4. Let $N \in \widetilde{\mathcal{G I}}$. Then for any R-module $M$ of finite injective or finite projective dimension,

$$
\partial_{\mathcal{G} \mathcal{I}}^{n}(M, N): \operatorname{ext}_{\mathcal{G} \mathcal{I}}^{n}(M, N) \rightarrow \operatorname{Ext}_{R}^{n}(M, N)
$$

is bijective for all $n \in \mathbb{Z}$.

Proof. The validity of the result is clear for $n \leq 0$. Let $n>0$. We induct on $n$. First note that for any G-injective $R$-module $E$, by definition $\operatorname{ext}_{\mathcal{G} \mathcal{I}}^{n}(M, E)=0$ for all $n>0$. Moreover by [EJ2, 10.1.3], $\operatorname{Ext}_{R}^{n}(M, E)=0$ for all $n>0$. Now suppose inductively that $n>0$ and consider short exact sequence

$$
0 \rightarrow N \rightarrow E \rightarrow L \rightarrow 0,
$$

where $E$ is G-injective and $\operatorname{id}_{R} L=t-1$; for the existence of such a complex see the paragraph after Theorem 3.9 below (see also [H2, 2.15]). By the induction hypothesis, $\partial_{\mathcal{G I}}^{n-1}(M, E)$ and $\partial_{\mathcal{G} \mathcal{I}}^{n-1}(M, L)$ are both bijective. So the result follows by using the long exact sequence of the above theorem, the Five-Lemma and the usual long exact sequence of ' $\mathrm{Ext}_{R}$ '.

Definition 3.5. By a complete coresolution of $N$, we mean a diagram $N \stackrel{i}{\rightarrow} \mathbf{I} \stackrel{v}{\rightarrow} \mathbf{T}$, where $N \stackrel{i}{\rightarrow} \mathbf{I}$ is an $\mathcal{I}$-coresolution, $\mathbf{T}$ is a complex with the property that $T_{n} \in \mathcal{I}$ and $\mathrm{H}_{n}(\mathbf{T})=\mathrm{H}_{n} \operatorname{Hom}_{R}(I, \mathbf{T})=0$, for all $n \in \mathbb{Z}$ and all $I \in \mathcal{I}, v$ is a morphism, and $v_{n}$ is bijective for all $n \ll 0$.

Remark 3.6. Let $N$ be an $R$-module of finite G-injective dimension $n$. So the $n$th cosyzygy $\Omega^{n} N$ of an injective coresolution $\mathbf{I}$ of $N$ lies in $\mathcal{G} \mathcal{I}$. The injective coresolution $\mathbf{I}$ of $N$ and the complete coresolution $\mathbf{T}$ of $\Omega^{n} N$ truncated in degree 0 can be put together into a commutative pitchfork diagram

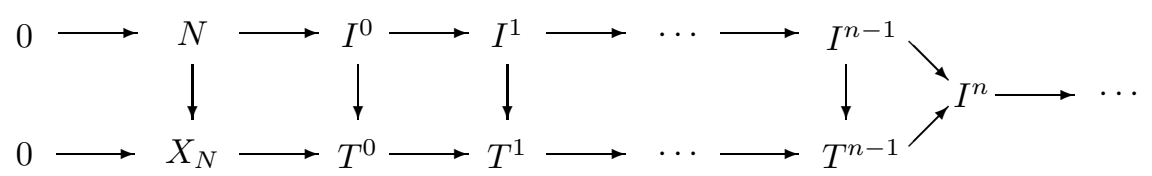

One can inductively construct the vertical maps, starting with the isomorphism $\Omega^{n} X_{N} \cong \Omega^{n} N$. So we have a map $f: N \rightarrow X_{N}$. Now we can lift this map to a chain map $v: \mathbf{I} \rightarrow \mathbf{T}$ (without loss of generality we may assume that $v$ is monomorphism). Using this we get a complete coresolution for $N$. As a result, we can record the following theorem.

Theorem 3.7. Let $N$ be an R-module. The following conditions are equivalent:

i) $\operatorname{Gid}_{R} N<\infty$.

ii) $N$ has a complete coresolution $N \stackrel{i}{\rightarrow} \mathbf{E} \stackrel{v}{\rightarrow} \mathbf{T}$ with $v_{n}$ bijective for all $n \gg 0$. 
Now suppose $N$ is an $R$-module of finite G-injective dimension. Choose a complete coresolution $N \stackrel{i}{\rightarrow} \mathbf{E} \stackrel{v}{\rightarrow} \mathbf{T}$. Let $M$ be an $R$-module. For any $n \in \mathbb{Z}$, define a Tate cohomology group by the equality

$$
\widehat{\operatorname{ext}}_{R}^{n}(M, N)=\mathrm{H}^{n} \operatorname{Hom}_{R}(M, \mathbf{T}) .
$$

By using the argument dual to that in the proof of [AM, 5.3] and then AM, 5.2] step by step, one can show that $\widehat{\operatorname{ext}}_{R}^{n}: \mathcal{C}(R) \times \widetilde{\mathcal{G I}}(R) \rightarrow \mathcal{C}(\mathbb{Z})$ is a functor and that there is a morphism of functors $\partial_{R}^{n}: \operatorname{Ext}_{R}^{n} \rightarrow \widehat{\operatorname{ext}}_{R}^{n}$ such that $\widehat{\operatorname{ext}}_{R}^{n}$ and $\varepsilon_{R}^{n}$ are independent of the choice of coresolutions and lifting. Moreover if $\operatorname{id}_{R} N$ is finite, by choosing a minimal injective coresolution $\mathbf{I}$ of $N$, one can construct a complete coresolution $N \rightarrow \mathbf{I} \rightarrow 0$, and so deduce that $\widehat{\operatorname{ext}}_{R}^{n}(, N)=0$ for all $n \in \mathbb{Z}$.

We have the same result for the case $\operatorname{id}_{R} M<\infty$. To see this let $N \stackrel{i}{\rightarrow} \mathbf{E} \stackrel{v}{\rightarrow} \mathbf{T}$ be a complete coresolution of $N$ and look at $\mathbf{T}$. Every (co)syzygy of $\mathbf{T}$ is G-injective. So [EJ2, 10.1.3] implies the result, i.e. $\widehat{\operatorname{ext}}_{R}^{n}(M, N)=0$ for all $n \in \mathbb{Z}$.

Dual statements to $\mathrm{AM}, 5.4$ and 5.6] also hold. That is:

Theorem 3.8. (a) Let $N$ be an R-module of finite $G$-injective dimension and let $0 \rightarrow M \rightarrow M^{\prime} \rightarrow M^{\prime \prime} \rightarrow 0$ be an exact sequence of $R$-modules. Then there exists a long exact sequences of Tate cohomology groups,

$$
\begin{gathered}
\cdots \longrightarrow \widehat{\operatorname{ext}}_{R}^{n}\left(M^{\prime \prime}, N\right) \longrightarrow \widehat{\operatorname{ext}}_{R}^{n}\left(M^{\prime}, N\right) \longrightarrow \widehat{\operatorname{ext}}_{R}^{n}(M, N) \\
\longrightarrow \widehat{\operatorname{ext}}_{R}^{n+1}\left(M^{\prime \prime}, N\right) \longrightarrow .
\end{gathered}
$$

(b) Let $0 \rightarrow N \rightarrow N^{\prime} \rightarrow N^{\prime \prime} \rightarrow 0$ be an exact sequence of $R$-modules of finite $G$-injective dimension and let $M$ be an arbitrary $R$-module. Then there exists a long exact sequence of Tate cohomology groups,

$$
\begin{gathered}
\cdots \longrightarrow \widehat{\operatorname{ext}}_{R}^{n}(M, N) \longrightarrow \widehat{\operatorname{ext}}_{R}^{n}\left(M, N^{\prime}\right) \longrightarrow \widehat{\operatorname{ext}}_{R}^{n}\left(M, N^{\prime \prime}\right) \\
\longrightarrow \widehat{\operatorname{ext}}_{R}^{n+1}(M, N) \longrightarrow .
\end{gathered}
$$

In both cases the connecting maps are natural in homomorphisms of $M$ and $N$.

The Tate cohomology theory is rigid in the sense that vanishing of any one of these functors implies the vanishing of all of them and their vanishing characterizes modules of finite injective dimension. We have in hand all the necessary materials to show this. We just mention that for any $R$-module $N$ of finite G-injective dimension, there exists a short exact sequence $0 \rightarrow N \rightarrow X \rightarrow L \rightarrow 0$ of $R$-modules such that $X$ is G-injective and $\operatorname{Gid}_{R} L=\operatorname{id}_{R} L=\operatorname{Gid}_{R} N-1$; see the paragraph after Theorem 3.9 below.

Now it is straightforward to prove the following result by using the same arguments as in [AM, 5.9].

Theorem 3.9. Let $N$ be an $R$-module with $\operatorname{Gid}_{R} N<\infty$. The following conditions are equivalent:

i) $\operatorname{id}_{R} N<\infty$.

ii) $\widehat{\operatorname{ext}}_{R}^{n}(, N)=0$, for some $n \in \mathbb{Z}$.

iii) $\widehat{\operatorname{ext}}_{R}^{n}(, N)=0$, for all $n \in \mathbb{Z}$.

iv) $\widehat{\operatorname{ext}}_{R}^{n}(N)=$,0 , for some $n \in \mathbb{Z}$.

v) $\widehat{\operatorname{ext}}_{R}^{n}(N)=$,0 , for all $n \in \mathbb{Z}$. 
Up to now the three cohomology theories - absolute, relative and Tate - are defined on the category $\widetilde{\mathcal{G I}}$ of modules of finite G-injective dimension. Relative and Tate functors come equipped with natural transformations

$$
\partial_{\mathcal{G I}}^{n}: \operatorname{ext}_{\mathcal{G I}}^{n} \rightarrow \operatorname{Ext}_{R}^{n} \text { and } \partial_{R}^{n}: \operatorname{Ext}_{R}^{n} \rightarrow \widehat{\operatorname{ext}}_{R}^{n} \text {. }
$$

Our last result in this section provides a tight connection between these three theories. Suppose that $\operatorname{Gid}_{R} N=n$. Using the pitchfork construction of Remark 3.6 we have a short exact sequence of complexes

$$
0 \rightarrow \mathbf{I} \stackrel{v}{\rightarrow} \mathbf{T} \rightarrow \mathbf{L} \rightarrow 0
$$

where $\mathbf{L}=\operatorname{Coker}(\mathbf{I} \rightarrow \mathbf{T})$. The long exact sequence of cohomology modules shows that the cohomology of $\mathbf{L}$ is concentrated in degree zero. Since $v$ is split in each degree, $\mathbf{L}$ is a complex of injective modules. Moreover since $v_{n}$ is the identity map for degrees bigger than or equal to $n$, terms of $\mathbf{L}$ are zero in all degrees bigger than or equal to $n$. So $\mathbf{L}$ is an injective coresolution of the module $Y_{N}$ defined by the short exact sequence

$$
o \rightarrow N \stackrel{f}{\rightarrow} X_{N} \rightarrow Y_{N} \rightarrow 0 .
$$

Since $\operatorname{id}_{R} Y_{N}<\infty$, this sequence is $\mathcal{G} \mathcal{I}$-proper. Furthermore, since $X_{N}$ is a cosyzygy of a complete resolution, it is G-injective. Thus the above short exact sequence is a $\mathcal{G I}$-proper coresolution of $N$. Moreover we can construct the exact sequence

$$
0 \rightarrow \mathbf{I} \stackrel{v^{b}}{\rightarrow} \mathbf{T}^{b} \stackrel{\chi^{b}}{\rightarrow} \Sigma^{-1} \mathbf{G} \rightarrow 0
$$

of complexes, where

$$
T_{n}^{b}=\left\{\begin{array}{ll}
T_{n} & \text { for } n \leq 0 ; \\
\Omega^{0} \mathbf{T} & \text { for } n=1 ; \\
0 & \text { for } n \geq 2 ;
\end{array} \quad \text { and } \quad G_{n}= \begin{cases}L_{n-1} & \text { for } n \leq-1 \\
\Omega^{0} \mathbf{T} & \text { for } n=0 \\
0 & \text { for } n \geq 1\end{cases}\right.
$$

The maps are defined in a manner similar to that in AM, 3.8]. Moreover $\gamma$ : $N \rightarrow \mathbf{G}$ with $\gamma_{0}: N \rightarrow \Omega^{0} \mathbf{T}$, is a strict $\mathcal{G} \mathcal{I}$-resolution of $N$. This sequence is split exact in each degree, so we have an exact sequence

$$
0 \longrightarrow \operatorname{Hom}_{R}(M, \mathbf{I}) \longrightarrow \operatorname{Hom}_{R}\left(M, \mathbf{T}^{b}\right) \longrightarrow \operatorname{Hom}_{R}\left(M, \Sigma^{-1} \mathbf{G}\right) \longrightarrow 0
$$

of complexes. This, in turn induces an exact sequence of cohomology modules as

$$
\begin{aligned}
\cdots & \rightarrow \mathrm{H}^{n} \operatorname{Hom}_{R}\left(M, \mathbf{T}^{b}\right) \rightarrow \mathrm{H}^{n} \operatorname{Hom}_{R}\left(M, \Sigma^{-1} \mathbf{G}\right) \rightarrow \mathrm{H}^{n+1} \operatorname{Hom}_{R}(M, \mathbf{I}) \\
& \rightarrow \mathrm{H}^{n+1} \operatorname{Hom}_{R}\left(M, \mathbf{T}^{b}\right) \rightarrow \cdots .
\end{aligned}
$$

Now one should identify the modules and maps appearing in this sequence to obtain the following theorem.

Theorem 3.10. Let $N$ be an R-module with $\operatorname{Gid}_{R} N=d<\infty$. Then for any $R$-module $M$, there exist natural homomorphisms $\gamma_{R}^{n}(M, N)$ in $M$ and in $N$, such that the following sequence is exact:

$$
\begin{aligned}
& 0 \longrightarrow \operatorname{ext}_{\mathcal{G} \mathcal{I}}^{1}(M, N) \stackrel{\partial_{\mathcal{G} \mathcal{I}}^{1}(M, N)}{\longrightarrow} \operatorname{Ext}_{R}^{1}(M, N) \longrightarrow \quad \ldots \\
& \longrightarrow \operatorname{ext}_{\mathcal{G} \mathcal{I}}^{n}(M, N) \stackrel{\partial_{\mathcal{G} \mathcal{I}}^{n}(M, N)}{\longrightarrow} \operatorname{Ext}_{R}^{n}(M, N) \stackrel{\partial_{R}^{n}(M, N)}{\longrightarrow} \widehat{\operatorname{ext}}_{R}^{n}(M, N) \\
& \stackrel{\gamma_{R}^{n}(M, N)}{\longrightarrow} \operatorname{ext}_{\mathcal{G I}}^{n+1}(M, N) \longrightarrow \quad \cdots \quad \longrightarrow \widehat{\operatorname{ext}}_{R}^{d}(M, N) \longrightarrow 0
\end{aligned}
$$

Corollary 3.11. Let $N$ be an $R$-module such that $\operatorname{Gid}_{R} N$ is finite. The following conditions are equivalent:

i) $\partial_{\mathcal{G} \mathcal{I}}^{n}(, N): \operatorname{ext}_{\mathcal{G} \mathcal{I}}^{n}(, N) \rightarrow \operatorname{Ext}_{R}^{n}(, N)$ is an isomorphism for all $n \in \mathbb{Z}$. 
ii) $\partial_{\mathcal{G I}}^{n}(N):, \operatorname{ext}_{\mathcal{G} \mathcal{I}}^{n}(N,) \rightarrow \operatorname{Ext}_{R}^{n}(N$,$) is an isomorphism for all n \in \mathbb{Z}$.

iii) $\operatorname{id}_{R} N<\infty$.

Proof. When $\operatorname{id}_{R} N$ is finite, $\widehat{\operatorname{ext}}_{R}^{n}(N)=,\widehat{\operatorname{ext}}_{R}^{n}(, N)=0$, for all $n \in \mathbb{Z}$. So the result follows from the previous long exact sequence.

\section{Balance of Gorenstein and Tate Derived Functors}

In this section we focus our attention on the relation between the notion of ' $\mathrm{Ext}_{\mathcal{G}}$ ' (resp. ' $\widehat{\operatorname{Ext}}_{R}$ ') and our definition of 'ext $\mathcal{G I}^{\prime}$ ' (resp. ' $\widehat{\operatorname{ext}}_{R}$ '). We deal with the balance of these functors over different subcategories of $\mathcal{C}$. We were told by the referee that the question of Gorenstein balance has already considered by Holm [H1. Actually he proved Theorem 4.3 (see [H1]).

Let us begin by recalling the notion of a $(\mathcal{G})$-relative cohomology. For any finitely generated module $M$ over a Noetherian ring $R$ that admits some $(\mathcal{G}$-)proper resolution, i.e. a resolution of $M$ by modules $G$ in the G-class, where $\operatorname{Hom}_{R}(G$, ) leaves the resolution exact for any module $G$ in $\mathrm{G}(R)$, Avramov and Martsinkovsky have [AM, Sec. 4] defined for each $n \in \mathbb{Z}$ and each $R$-module $N$ a relative cohomology group

$$
\operatorname{Ext}_{\mathcal{G}}^{n}(M, N)=H^{n} \operatorname{Hom}_{R}(\mathbf{G}, N),
$$

where $\mathbf{G} \rightarrow M$ is a $\mathcal{G}$-proper resolution. Choosing a projective resolution $\pi: \mathbf{P} \rightarrow$ $M$ and a morphism of complexes $\phi: \mathbf{P} \rightarrow \mathbf{G}$ that lifts the identity map of $M$, they defined the comparison morphism

$$
\varepsilon_{\mathcal{G}}^{n}(M, N): \operatorname{Ext}_{\mathcal{G}}^{n}(M, N) \rightarrow \operatorname{Ext}_{R}^{n}(M, N)
$$

by setting $\varepsilon_{\mathcal{G}}^{n}(M, N)=\mathrm{H}^{n} \operatorname{Hom}_{R}(\phi, N): \mathrm{H}^{n} \operatorname{Hom}_{R}(\mathbf{G}, N) \rightarrow \mathrm{H}^{n} \operatorname{Hom}_{R}(\mathbf{P}, N)$. Moreover, they showed that $\operatorname{Ext}_{\mathcal{G}}^{n}$ and $\varepsilon_{\mathcal{G}}^{n}$ are independent of the choice of resolutions and liftings AM, 4.2].

It follows from [EJ2, 12.1.4] that over Gorenstein rings of finite dimension, for any $R$-modules $M$ and $N$ and any $i \in \mathbb{Z}$ we have

$$
\operatorname{Gext}_{R}^{i}(M, N)=\operatorname{Ext}_{\mathcal{G}}^{i}(M, N)=\operatorname{ext}_{\mathcal{G} \mathcal{I}}^{i}(M, N) .
$$

Lemma 4.1. Let $M \in \widetilde{\mathcal{G}}$. Then any $\mathcal{G}$-proper resolution $\mathbf{G} \rightarrow M$ is $\operatorname{Hom}_{R}(, \mathcal{G I})$ exact.

Proof. Let $0 \rightarrow X \rightarrow G_{0} \rightarrow M \rightarrow 0$ be a $\mathcal{G}$-approximation of $M$, i.e. $G_{0} \in$ $\mathrm{G}(R), \operatorname{pd}_{R} X<\infty$ and the sequence is exact; for the existence of such a sequence see [AM, 3.1]. Let $E \in \mathcal{G I}$. It is enough to show that this sequence is $\operatorname{Hom}_{R}(, E)$ exact. By $\mathrm{AM}, 4.6]$, there exists an exact sequence

$$
0 \rightarrow \operatorname{Hom}_{R}(M, E) \rightarrow \operatorname{Hom}_{R}\left(G_{0}, E\right) \rightarrow \operatorname{Hom}_{R}(X, E) \rightarrow \operatorname{Ext}_{\mathcal{G}}^{1}(M, E) .
$$

So it is enough to prove that $\operatorname{Ext}_{\mathcal{G}}^{1}(M, E)=0$. To see this, for any integer $i \geq 0$ consider the short exact sequences

$$
0 \rightarrow L_{i} \rightarrow I_{i} \rightarrow L_{i-1} \rightarrow 0
$$


whose middle terms are injective. They exist because of the G-injectivity of $E$. Here $L_{-1}=E$. So for any integer $i>0$, we get the following commutative diagram:

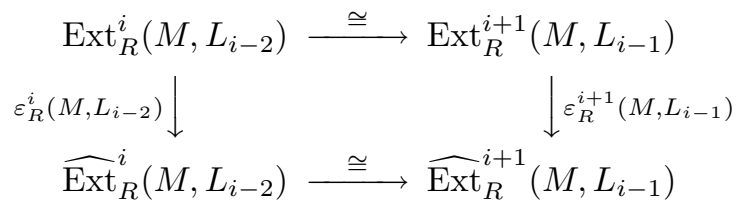

But for $i>\mathrm{G}$ - $\operatorname{dim}_{R} M, \varepsilon_{R}^{i}\left(M, L_{i-2}\right)$ is an isomorphism. As a result we get the isomorphism of $\varepsilon_{R}^{1}(M, E): \operatorname{Ext}_{R}^{1}(M, E) \rightarrow \widehat{\operatorname{Ext}}_{R}^{1}(M, E)$, which in conjunction with [AM, 7.1] implies that $\operatorname{Ext}_{\mathcal{G}}^{1}(M, E)=0$. This completes the proof.

Lemma 4.2. Let $N \in \widetilde{\mathcal{G I}}$. Then any $\mathcal{G I}$-proper coresolution $N \rightarrow \mathbf{E}$ of $N$ is $\operatorname{Hom}_{R}(\mathcal{G}$,$) -exact.$

Proof. Dual the proof of the above lemma.

The above two lemmas can be stated in the language of Enochs and Jenda EJ2, 8.2.13] as follows: The bifunctor $\operatorname{Hom}_{R}($,$) is right balanced by \widetilde{\mathcal{G}} \times \widetilde{\mathcal{G I}}$. So we have the following nice result.

Theorem 4.3. Let $M \in \widetilde{\mathcal{G}}$ and $N \in \widetilde{\mathcal{G I}}$. Then for all $i \in \mathbb{Z}$,

$$
\operatorname{ext}_{\mathcal{G} \mathcal{I}}^{i}(M, N) \cong \operatorname{Ext}_{\mathcal{G}}^{i}(M, N) \text {. }
$$

Proof. Use [EJ2, 8.2.14].

Over Gorenstein rings we can generalize the above result to $\mathcal{F} \times \overline{\mathcal{G I}}$, where $\mathcal{F}$ denotes the full subcategory of finite $R$-modules. To this end we need some preliminary results.

Lemma 4.4. Let $G$ be a $G$-injective $R_{\mathfrak{p}}$-module, where $\mathfrak{p}$ is a prime ideal of $R$. Then $G$ is also $G$-injective as an $R$-module.

Proof. By definition there exists a complex $\mathbf{T}$ of injective $R_{\mathfrak{p}}$-modules with the property that $\mathrm{H}_{n}(\mathbf{T})=\mathrm{H}_{n}\left(\operatorname{Hom}_{R_{\mathfrak{p}}}(I, \mathbf{T})\right)=0$ for all $n \in \mathbb{Z}$ and all injective $R_{\mathfrak{p}}$ module $I$ and $G$ is a cosyzygy of this complex. Clearly the terms of this complex are also injective as $R$-modules. Let $I$ be an injective $R$-module. We have to show that $\mathrm{H}_{n}\left(\operatorname{Hom}_{R}(I, \mathbf{T})\right)=0$. Since $\mathbf{T}$ is a complex of $R_{\mathfrak{p}}$-modules, $\operatorname{Hom}_{R}(I, \mathbf{T}) \cong \operatorname{Hom}_{R_{\mathfrak{p}}}\left(I_{\mathfrak{p}}, \mathbf{T}\right)$. Moreover $I_{\mathfrak{p}}$ is injective $R_{\mathfrak{p}}$-module. So the result follows.

Proposition 4.5. Let $M$ be a finitely generated $R$-module and $N \in \overline{\mathcal{G I}}$. Then for all $\mathfrak{p} \in \operatorname{Spec}(R)$ and for all $i \geq 0$, there exist isomorphisms

$$
\left(\operatorname{ext}_{\mathcal{G I}}^{i}(M, N)\right)_{\mathfrak{p}} \cong \operatorname{ext}_{\mathcal{G} \mathcal{I}_{\mathfrak{p}}}^{i}\left(M_{\mathfrak{p}}, N_{\mathfrak{p}}\right)
$$

where $\mathcal{G I}_{\mathfrak{p}}$ is the class of all G-injective $R_{\mathfrak{p}}$-modules.

Proof. First we show that if $0 \rightarrow N \rightarrow \mathbf{E}$ is a $\mathcal{G} \mathcal{I}$-proper coresolution, then for any prime $\mathfrak{p}$ in $\operatorname{Spec}(R), 0 \rightarrow N_{\mathfrak{p}} \rightarrow \mathbf{E}_{\mathfrak{p}}$ is a $\mathcal{G} \mathcal{I}_{\mathfrak{p}}$-proper coresolution. It is enough to deal with the $\mathcal{G I}$-proper sequence $0 \rightarrow N \rightarrow E \rightarrow L \rightarrow 0$. Let $G$ be an arbitrary Ginjective $R_{\mathfrak{p}}$-module. We must show that the sequence $0 \rightarrow N_{\mathfrak{p}} \rightarrow E_{\mathfrak{p}} \rightarrow L_{\mathfrak{p}} \rightarrow 0$ is $\operatorname{Hom}_{R_{\mathfrak{p}}}(, G)$-exact. But since, by the previous lemma, $G$ is G-injective $R$-module, the original sequence (before localizing) is $\operatorname{Hom}_{R}(, G)$-exact. Now the result follows using the natural isomorphism $\operatorname{Hom}_{R}(, G) \cong \operatorname{Hom}_{R_{\mathfrak{p}}}\left(()_{\mathfrak{p}}, G\right)$. 
Corollary 4.6. Let $R$ be a (not necessarily finite-dimensional) Gorenstein ring. Let $G \in G(R)$ and $N \in \overline{\mathcal{G I}}$. Then $\operatorname{ext}_{\mathcal{G I}}^{1}(G, N)=0$.

Proof. Let $\mathfrak{p}$ be a prime ideal of $R$. The localized module $\operatorname{ext}_{\mathcal{G I}_{\mathfrak{p}}}^{1}\left(G_{\mathfrak{p}}, N_{\mathfrak{p}}\right)$ fulfills the conditions that $G_{\mathfrak{p}} \in \mathrm{G}\left(R_{\mathfrak{p}}\right)$ and $\operatorname{Gid}_{R_{\mathfrak{p}}} M_{\mathfrak{p}}<\infty$. So by Theorem 4.3, $\operatorname{ext}_{\mathcal{G I}_{\mathfrak{p}}}^{1}\left(G_{\mathfrak{p}}, N_{\mathfrak{p}}\right) \cong \operatorname{Ext}_{\mathcal{G}_{\mathfrak{p}}}^{1}\left(G_{\mathfrak{p}}, N_{\mathfrak{p}}\right)$. The latter module is zero by definition. This implies the result as $\mathfrak{p}$ was arbitrary.

Theorem 4.7. Let $R$ be a (not necessarily finite-dimensional) Gorenstein ring. Let $M$ be finitely generated and $N \in \overline{\mathcal{G I}}$. Then for all $i \in \mathbb{Z}$,

$$
\operatorname{ext}_{\mathcal{G I}}^{i}(M, N) \cong \operatorname{Ext}_{\mathcal{G}}^{i}(M, N) \text {. }
$$

Proof. The result holds trivially for $i \leq 0$. So we only have to deal with the case $i>0$. By [G], G- $\operatorname{dim}_{R} M$ is finite. So there are short exact sequences

$$
0 \longrightarrow L \longrightarrow G \longrightarrow M \longrightarrow 0
$$

and

$$
0 \longrightarrow N \longrightarrow E \longrightarrow K \longrightarrow 0,
$$

such that $G \in \mathrm{G}(R), E$ is G-injective and they are respectively $\mathcal{G}$ - and $\mathcal{G} \mathcal{I}$-proper. We have the following commutative diagram:

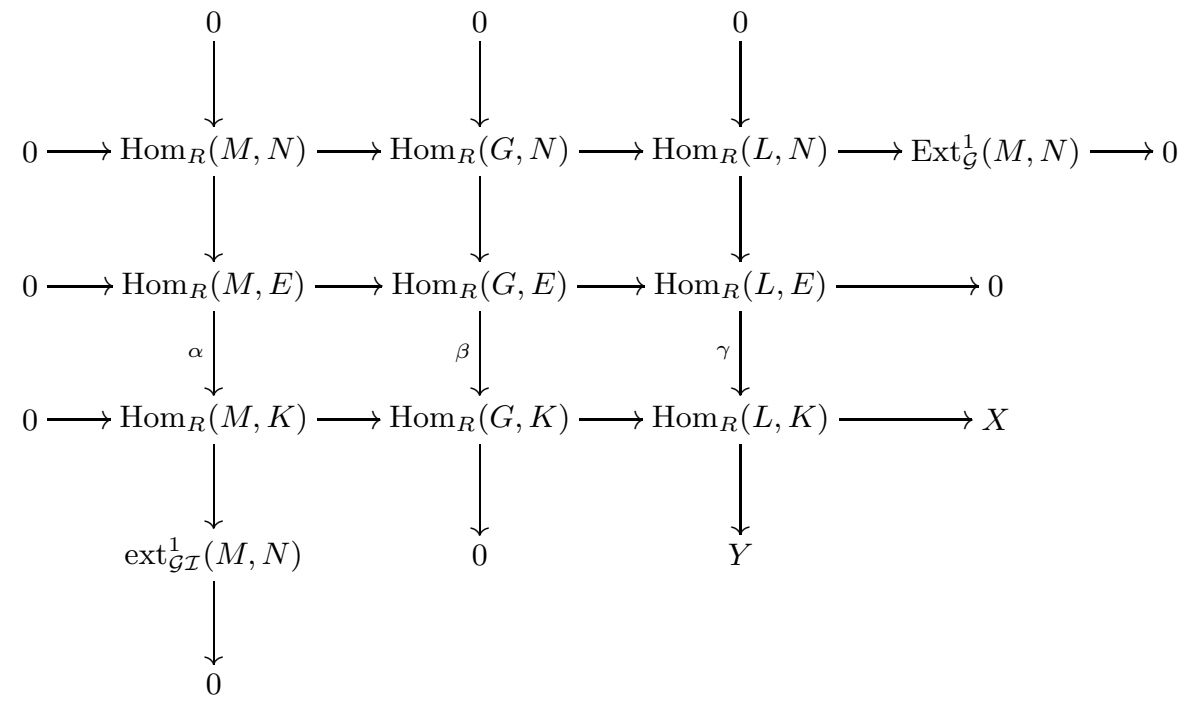

By Theorem 4.3, $\operatorname{Ext}_{\mathcal{G}}^{1}(M, E) \cong \operatorname{ext}_{\mathcal{G I}}^{1}(M, E)$ and so is zero by definition. This implies the exactness of the middle row. The exactness of the middle column follows from the previous corollary. Now applying the snake lemma (to the maps $\alpha, \beta$ and $\gamma$ ) yields an exact sequence

$$
\text { Ker } \beta \longrightarrow \operatorname{Ker} \gamma \longrightarrow \text { Coker } \alpha \longrightarrow \text { Coker } \beta,
$$

i.e.

$$
\operatorname{Hom}_{R}(G, N) \rightarrow \operatorname{Hom}_{R}(L, N) \rightarrow \operatorname{ext}_{\mathcal{G \mathcal { I }}}^{1}(M, N) \rightarrow 0 .
$$

Comparing this with the first row of the diagram gives the isomorphism

$$
\operatorname{Ext}_{\mathcal{G I}}^{1}(M, N) \cong \operatorname{ext}_{\mathcal{G} \mathcal{I}}^{1}(M, N)
$$


One can now use the argument of the proof of $[\underline{R}, 7.8]$ to get the isomorphism for all $n$.

We now want to give a sort of balance for Tate cohomology groups. Before doing so we quote the definition of $\widehat{\operatorname{Ext}}_{R}$ from [AM]. Let $\mathcal{P}$ denote the full subcategory of $\mathcal{C}$, whose objects are the finite projective $R$-modules. A complex $\mathbf{T}$ is called totally acyclic if $T_{n} \in \mathcal{P}$ and $\mathrm{H}_{n}(\mathbf{T})=0=\mathrm{H}_{n}\left(\operatorname{Hom}_{R}(\mathbf{T}, R)\right)$. Let $M$ be a finite $R$-module of finite G-dimension. Choose a complete resolution $\mathbf{T} \stackrel{v}{\rightarrow} \mathbf{P} \stackrel{\pi}{\rightarrow} M$ of $M$ [AM, 3.1]. By this we mean a diagram $\mathbf{T} \stackrel{v}{\rightarrow} \mathbf{P} \stackrel{\pi}{\rightarrow} M$, where $\pi$ is a $\mathcal{P}$-resolution of $M, \mathbf{T}$ is a totally acyclic complex, $v$ is a morphism, and $v_{n}$ is bijective for all $n \gg 0$. Then for each $R$-module $N$ and for each $n \in \mathbb{Z}$ a Tate cohomology group is defined by the equality

$$
\widehat{\operatorname{Ext}}_{R}^{n}(M, N)=\mathrm{H}^{n} \operatorname{Hom}_{R}(\mathbf{T}, N) .
$$

In [AM, Sec. 5], it is shown that the functors $\widehat{\operatorname{Ext}}_{R}^{n}$ are independent of the choice of resolutions. For more details see [AM, Sec. 5].

Theorem 4.8. Let $(R, \mathfrak{m})$ be a Gorenstein local ring. Let $M$ be a finite and let $N$ be an arbitrary $R$-module. Then for all $i \in \mathbb{Z}$,

$$
\widehat{\operatorname{Ext}}_{R}^{i}(M, N) \cong \widehat{\operatorname{ext}}_{R}^{i}(M, N) \text {. }
$$

Proof. Since $R$ is Gorenstein local, both G- $\operatorname{dim}_{R} M$ and $\operatorname{Gid}_{R} N$ are finite. Set $t=\mathrm{G}-\operatorname{dim}_{R} M$. By [AM, 7.1], Theorem 3.10 and Theorem 4.3, for all $i>t$ $\widehat{\operatorname{Ext}}_{R}^{i}(M, N) \cong \widehat{\operatorname{ext}}_{R}^{i}(M, N)$ since both modules are isomorphic to the ordinary 'Ext' modules. Let $i \in \mathbb{Z}$. Consider the short exact sequence

$$
0 \rightarrow L_{0} \rightarrow F_{0} \rightarrow N \rightarrow 0
$$

where $F_{0}$ is free. By $[\mathrm{AM}, 5.4]$, there is a long exact sequence

$$
\cdots \rightarrow \widehat{\operatorname{Ext}}_{R}^{i}\left(M, F_{0}\right) \rightarrow \widehat{\operatorname{Ext}}_{R}^{i}(M, N) \rightarrow \widehat{\operatorname{Ext}}_{R}^{i+1}\left(M, L_{0}\right) \rightarrow \widehat{\operatorname{Ext}}_{R}^{i+1}\left(M, F_{0}\right) \rightarrow \cdots
$$

Since $F_{0}$ is free, by [AM, 5.9], $\widehat{\operatorname{Ext}}_{R}^{i}\left(, F_{0}\right)=0$ for all $i \in \mathbb{Z}$. This implies the isomorphism $\widehat{\operatorname{Ext}}_{R}^{i}(M, N) \cong \widehat{\operatorname{Ext}}_{R}^{i+1}\left(M, L_{0}\right)$. Continuing in this way but now considering the exact sequence

$$
0 \rightarrow L_{1} \rightarrow F_{1} \rightarrow L_{0} \rightarrow 0
$$

we are able to increase the powers of $\widehat{\operatorname{Ext}}_{R}$ as far as we want, say up to $t+1$, where $\widehat{\operatorname{Ext}}_{R}$ is isomorphic to ordinary 'Ext'. Now again consider the sequence

$$
0 \rightarrow L_{0} \rightarrow F_{0} \rightarrow N \rightarrow 0
$$

and apply Theorem 3.8 to obtain the exact sequence

$$
\cdots \rightarrow \widehat{\operatorname{ext}}_{R}^{i}\left(M, F_{0}\right) \rightarrow \widehat{\operatorname{ext}}_{R}^{i}(M, N) \rightarrow \widehat{\operatorname{ext}}_{R}^{i+1}\left(M, L_{0}\right) \rightarrow \widehat{\operatorname{ext}}_{R}^{i+1}\left(M, F_{0}\right) \rightarrow \cdots
$$

The Gorensteinness of $R$ implies that $\operatorname{id}_{R} F_{0}<\infty$. So by Theorem 3.9, $\widehat{\operatorname{ext}}_{R}^{i}\left(, F_{0}\right)=$ 0 for all $i \in \mathbb{Z}$. This gives the isomorphism $\widehat{\operatorname{ext}}_{R}^{i}(M, N) \cong \widehat{\operatorname{ext}}_{R}^{i+1}\left(M, L_{0}\right)$. So again we can increase the superscript of $\widehat{\operatorname{ext}}_{R}$ up to $t+1$. The result now follows.

Remark 4.9. There is an unpublished cohomology theory due to Pierre Vogel that associates to each pair $(M, N)$ of modules over an arbitrary ring $R$ a sequence of abelian groups $\widetilde{\operatorname{Ext}}_{R}^{n}(M, N)$ for $n \in \mathbb{Z}$ and which comes equipped with a natural 
transformation $\operatorname{Ext}_{R}^{n}(M, N) \longrightarrow{\widetilde{\operatorname{Ext}_{R}}}_{R}^{n}(M, N)$ of cohomology functors. For more details see $\mathrm{M}$.

In the literature, there is another complete cohomology theory, using injectives. For details of the construction see e.g. [N]. In this theory injective complete coresolutions can be applied to define cohomology functors $\operatorname{Ext}_{R}^{*}($,$) which are also$ equipped with the natural maps $\operatorname{Ext}_{R}^{n}(M, N) \longrightarrow \operatorname{Ext}_{R}^{n}(M, N)$. When $R$ is a commutative Noetherian local ring, it is known [N, 5.2] that the (projective) complete cohomology $\widetilde{\operatorname{Ext}}_{R}^{*}(M, N)$ and the (injective) complete cohomology $\check{E x t}_{R}^{*}(M, N)$ are naturally equivalent if and only if $R$ is Gorenstein.

It is easy to see that if $N \in \widetilde{\mathcal{G I}}$, there is a natural isomorphism of cohomology functors $\operatorname{Ext}_{R}^{*}(M, N) \cong \widehat{\operatorname{ext}}_{R}^{*}(M, N)$ compatible with the natural maps from $\operatorname{Ext}_{R}^{n}(M, N)$. We observe that using the above theorem, for any finite module $M$ over Gorenstein ring of finite dimension, there is a natural isomorphism of cohomology functors

$$
\widehat{\operatorname{Ext}}_{R}^{*}(M, N) \cong \widetilde{\operatorname{Ext}}_{R}^{*}(M, N) \cong \tilde{\operatorname{Ext}}_{R}^{*}(M, N) \cong \widehat{\operatorname{ext}}_{R}^{*}(M, N)
$$

Remark 4.10. For a finite module $M$ over a Gorenstein complete local ring $(R, \mathfrak{m})$, Auslander defined the delta invariant $\delta_{R}(M)$ to be the smallest integer $n$ such that there is an epimorphism $X \oplus R^{n} \rightarrow M$ with $X$ a maximal Cohen-Macaulay module with no free summand. For an integer $i \geq 0$ he defined the $i$ th higher delta invariant $\delta_{R}^{i}(M)$ by the formula $\delta_{R}^{i}(M)=\delta_{R}\left(\Omega_{R}^{i}(M)\right)$, where $\Omega_{R}^{i}(M)$ denotes the $i$ th syzygy module in a minimal free resolution of $M$ over $R$; cf. [ADS, Sec. 5].

On the other hand, by [AM, 5.2] for any such module $M$, there exist natural maps $\varepsilon_{R}^{i}(M, k): \operatorname{Ext}_{R}^{i}(M, k) \longrightarrow \widehat{\operatorname{Ext}}_{R}^{i}(M, k)$, for any integer $i$. The $i$ th higher delta invariant can also be defined as the $\operatorname{dim}_{k} \operatorname{Ker}\left(\varepsilon_{R}^{i}(M, k)\right)$ (see [M] $)$.

In the following we show that these invariants can also be defined as the dimensions of the $k$-vector spaces $\operatorname{Ker}\left(\partial_{R}^{i}(M, k): \operatorname{Ext}_{R}^{i}(M, k) \rightarrow \widehat{\operatorname{ext}}_{R}^{i}(M, k)\right)$, of Theorem 3.10 .

Theorem 4.11. Let $(R, \mathfrak{m})$ be a Gorenstein local ring. Let $M$ be a finitely generated $R$-module. Then for any $i>0$,

$$
\delta_{R}^{i}(M)=\operatorname{dim}_{k} \operatorname{Ker}\left(\partial_{R}^{i}(M, k): \operatorname{Ext}_{R}^{i}(M, k) \rightarrow \widehat{\operatorname{ext}}_{R}^{i}(M, k)\right) .
$$

Proof. Let $X$ and $Y$ be two arbitrary $R$-modules with $X$ finitely generated. It follows from [AM, 7.1] that $\operatorname{dim}_{k} \operatorname{Ker}\left(\varepsilon_{R}^{1}(X, Y)\right)=\operatorname{dim}_{k} \operatorname{Ext}_{\mathcal{G}}^{1}(X, Y)$. On the other hand Theorem 3.10, implies that $\operatorname{Ker}\left(\partial_{R}^{1}(X, Y)\right)=\operatorname{ext}_{\mathcal{G I}}^{1}(X, Y)$. So use Theorem 4.3 to deduce that $\operatorname{Ker}\left(\varepsilon_{R}^{1}(X, Y)\right) \cong \operatorname{Ker}\left(\partial_{R}^{1}(X, Y)\right)$.

Now let $M$ be a finitely generated $R$-module. By the above paragraph, $\delta_{R}^{1}(M)=$ $\operatorname{Ker}\left(\partial_{R}^{1}(M, k)\right)$. Consider the short exact sequence $0 \rightarrow L \rightarrow F \rightarrow M \rightarrow 0$, where $F$ is free and G- $\operatorname{dim}_{R} L$ is finite. Since $R$ is a Gorenstein local ring, $\operatorname{id}_{R} F$ is finite. So we have the following commutative diagrams:
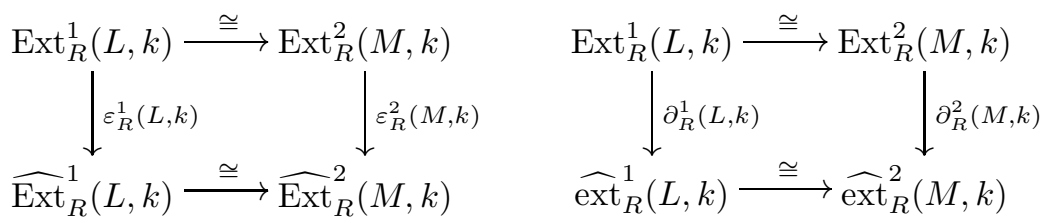
They in turn, imply that

$$
\delta_{R}^{2}(M)=\operatorname{dim}_{k} \operatorname{Ker}\left(\varepsilon_{R}^{1}(L, k)\right)=\operatorname{dim}_{k} \operatorname{Ker}\left(\partial_{R}^{1}(L, k)\right)=\operatorname{dim}_{k} \operatorname{Ker}\left(\partial_{R}^{2}(M, k)\right) .
$$

The same argument shows that $\delta_{R}^{2}(L)=\operatorname{dim}_{k} \operatorname{Ker}\left(\partial_{R}^{2}(L, k)\right)$. Now use induction.

Corollary 4.12. Let $R$ be a Gorenstein local ring. Then $R$ is regular if $\operatorname{Ker} \partial_{R}^{i}(k, k)$ $\neq 0$ for some $i>0$.

Proof. In view of the above corollary the result follows from [ADS, 5.7] .

\section{Gorenstein LOCAL COHOMOLOGY MODUles}

The main objec of this section is to introduce and study a generalization of local cohomology functors which were originally introduced by Grothendieck in local algebra. The local cohomology functors are defined as the right derived functors of the appropriate torsion functor and it can be shown that one can construct them as direct limit of 'Ext' modules. We follow this scheme and introduce the Gorenstein local cohomology modules. To begin we recall that for an $R$-module $M$, the atorsion functor is defined as $\Gamma_{\mathfrak{a}}(M)=\bigcup_{n \in \mathbb{N}}\left(0::_{M} \mathfrak{a}^{n}\right)$. It is naturally equivalent to the functor $\lim \operatorname{Hom}_{R}\left(R / \mathfrak{a}^{n},\right)$.

$$
\overrightarrow{n \in \mathbb{N}}
$$

Definition 5.1. Let $M$ be an $R$-module and let $\mathfrak{a}$ be an ideal of $R$. Choose $N \in \overline{\mathcal{G I}}$ and take a $\mathcal{G} \mathcal{I}$-proper coresolution $N \rightarrow \mathbf{E}$. Apply the functor $\operatorname{Hom}_{R}\left(M, \Gamma_{\mathfrak{a}}()\right)$ on it. Take the $i$ th cohomology module of the latter complex; the result

$$
\frac{\operatorname{Ker}\left(\operatorname{Hom}_{R}\left(M, \Gamma_{\mathfrak{a}}\left(\partial_{i}\right)\right)\right)}{\operatorname{Im}\left(\operatorname{Hom}_{R}\left(M, \Gamma_{\mathfrak{a}}\left(\partial_{i+1}\right)\right)\right)}
$$

which, using standard facts of homological algebra, is independent (up to $R$-isomorphism) of the choice of $\mathcal{G} \mathcal{I}$-proper coresolutions of $M$, will be denoted by $G H_{\mathfrak{a}}^{i}(M, N)$ and will be referred as the $i$ th Gorenstein local cohomology module of $M$ and $N$ with respect to $\mathfrak{a}$.

Since $\operatorname{Hom}_{R}\left(M, \Gamma_{\mathfrak{a}}()\right)$ is left exact, $G H_{\mathfrak{a}}^{0}(M, \quad)$ is naturally equivalent to $\operatorname{Hom}_{R}\left(M, \Gamma_{\mathfrak{a}}()\right)$. So we can use this natural equivalence to identify two functors. Moreover using standard tools in homological algebra, it is not difficult to see that we can consider $G H_{\mathfrak{a}}^{i}(M, N)$ as a direct limit of the $\operatorname{ext}_{\mathcal{G} \mathcal{I}}^{i}$. More precisely, for any $i \in \mathbb{Z}$, there is an isomorphism

$$
G H_{\mathfrak{a}}^{i}(M, N) \cong \underset{n \in \mathbb{N}}{\lim } \operatorname{ext}_{\mathcal{G} \mathcal{I}}^{i}\left(M / \mathfrak{a}^{n} M, N\right) .
$$

Lemma 5.2. Let $M$ be a G-injective $R$-module. Then for any ideal $\mathfrak{a}$ of $R, M$ is $\Gamma_{\mathfrak{a}}$-acyclic, that is, $H_{\mathfrak{a}}^{i}(M)=0$ for all $i>0$.

Proof. Let $M$ be a G-injective module. By definition, for any integer $i \geq 0$, there exist short exact sequences

$$
0 \rightarrow L_{i} \rightarrow E_{i} \rightarrow L_{i-1} \rightarrow 0
$$

where the $E_{i}$ 's are injective and $L_{-1}=M$. Applying the a-torsion functor $\Gamma_{\mathfrak{a}}$ on these exact sequences, in conjunction with the fact that for any injective module $I$, $H_{\mathfrak{a}}^{i}(I)=0$ for all $i>0$, implies a sequences of isomorphisms

$$
H_{\mathfrak{a}}^{1}(M) \cong H_{\mathfrak{a}}^{2}\left(L_{0}\right) \cong \cdots \cong H_{\mathfrak{a}}^{i}\left(L_{i-2}\right) \cong \cdots .
$$


Since $R$ is Noetherian, the arithmetic rank of $\mathfrak{a}$, ara $(\mathfrak{a}=t)$ is finite. So by BS, 3.3.3], for all $i>0, H_{\mathfrak{a}}^{i+t}\left(L_{i-2}\right)=0$. So the result follows.

Remark 5.3. ( $i$ ). As explained in [BS, 1.2.2], the most basic method for calculation of $H_{\mathfrak{a}}^{i}(N)$ for an $R$-module $N$ is to take an injective coresolution $\mathbf{I}^{\bullet}$ of $N$, apply $\Gamma_{\mathfrak{a}}$ (the $\mathfrak{a}$-torsion functor) to $\mathbf{I}^{\bullet}$ to obtain the complex $\Gamma_{\mathfrak{a}}\left(\mathbf{I}^{\bullet}\right)$, and take the $i$ th cohomology module of this complex: we have $H_{\mathfrak{a}}^{i}(N)=H^{i}\left(\Gamma_{\mathfrak{a}}\left(\mathbf{I}^{\bullet}\right)\right)$.

It is an easy exercise in homological algebra to see that a coresolution of $N$ by $\Gamma_{\mathfrak{a}}$-acyclic $R$-modules will serve this purpose just as well. So by the previous lemma one can compute local cohomology modules using a $\mathcal{G} \mathcal{I}$-coresolution of $N$.

This fact shows that $G H_{\mathfrak{a}}^{i}(R, N)=H_{\mathfrak{a}}^{i}(N)$ for all $i \in \mathbb{Z}$. So our definition is in fact a generalization of usual local cohomology functor.

(ii). There is a generalization of local cohomology functors given by Herzog in He. For each $i \geq 0, H_{\mathfrak{a}}^{i}():, \mathcal{C} \times \mathcal{C} \rightarrow \mathcal{C}$ is the functor defined by $H_{\mathfrak{a}}^{i}(M, N)=$ $\lim \operatorname{Ext}_{R}^{i}\left(\frac{M}{\mathfrak{a}^{n} M}, N\right)$, where $M, N \in \mathcal{C}$. It is clear that the functor $H_{\mathfrak{a}}^{i}(R$,$) is naturally$ $\overrightarrow{n \in \mathbb{N}}$

equivalent to the functor $H_{\mathfrak{a}}^{i}()$.

If $N$ is an $R$-module of finite injective dimension, then every injective coresolution of $N$ is a $\mathcal{G I}$-proper coresolution EJ2, 10.1.3]. So in this case for any $R$-module $M, G H_{\mathfrak{a}}^{i}(M, N)=H_{\mathfrak{a}}^{i}(M, N)$, for all $i \in \mathbb{Z}$.

Theorem 5.4. Let $0 \rightarrow N \rightarrow N^{\prime} \rightarrow N^{\prime \prime} \rightarrow 0$ be a $\mathcal{G I}$-proper exact sequence of $R$-modules in $\overline{\mathcal{G I}}$. Then for any $R$-module $M$, there is a long exact sequence of Gorenstein local cohomology modules as follows:

$$
\begin{gathered}
0 \longrightarrow G H_{\mathfrak{a}}^{0}(M, N) \longrightarrow G H_{\mathfrak{a}}^{0}\left(M, N^{\prime}\right) \longrightarrow G H_{\mathfrak{a}}^{0}\left(M, N^{\prime \prime}\right) \\
\left.\longrightarrow G H_{\mathfrak{a}}^{1}(M, N) \longrightarrow N^{\prime}\right) \longrightarrow .
\end{gathered}
$$

Proof. The functor $\operatorname{Hom}_{R}\left(M, \Gamma_{\mathfrak{a}}()\right)$ is a covariant left exact functor. So the result follows from [EJ2, 8.2.5].

Lemma 5.5. Let $M$ and $E$ be $R$-modules such that $\operatorname{pd}_{R} M$ is finite and $E$ is $G$-injective. Then for any $i>0, H_{\mathfrak{a}}^{i}(M, E)=0$.

Proof. Since $E$ is G-injective, for any integer $t \geq 0$, there exists exact sequence

$$
0 \longrightarrow L_{t} \longrightarrow I_{t} \longrightarrow L_{t-1} \longrightarrow 0
$$

where the middle terms are all injective and $L_{-1}=E$. Using them, for any $i \in \mathbb{N}$, we obtain a sequence of isomorphisms as follows:

$$
H_{\mathfrak{a}}^{i}(M, E) \cong H_{\mathfrak{a}}^{i+1}\left(M, L_{0}\right) \cong H_{\mathfrak{a}}^{i+2}\left(M, L_{1}\right) \cong \cdots
$$

By [B] the generalized local cohomology module will vanish after $\operatorname{pd}_{R} M+\operatorname{ara}(\mathfrak{a})$. So the result follows.

Proposition 5.6. Let $\operatorname{pd}_{R} M$ be finite and let $N \in \overline{\mathcal{G I}}$. Then for any $i \in \mathbb{Z}$,

$$
G H_{\mathfrak{a}}^{i}(M, N) \cong H_{\mathfrak{a}}^{i}(M, N) .
$$

Proof. There exists a $\mathcal{G} \mathcal{I}$-proper exact sequence of $R$-modules

$$
0 \longrightarrow N \longrightarrow E \longrightarrow L \longrightarrow 0,
$$

where $E$ is $\mathrm{G}$-injective and $L \in \overline{\mathcal{G I}}$. So, in view of the previous lemma, we obtain 
a commutative diagram

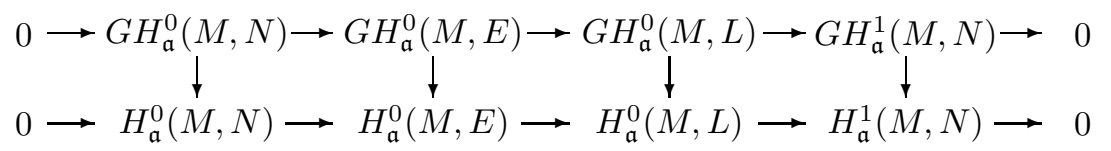

(in which the rows are exact), and for each $i \in \mathbb{N}$, isomorphisms $G H_{\mathfrak{a}}^{i}(M, L) \cong$ $G H_{\mathfrak{a}}^{i+1}(M, N)$ and $H_{\mathfrak{a}}^{i}(M, L) \cong H_{\mathfrak{a}}^{i+1}(M, N)$. Since the first three vertical maps are isomorphisms, so is the fourth. Since $L$ also belongs to $\overline{\mathcal{G}} \overline{\mathcal{I}}$, the same arguments imply the isomorphism $G H_{\mathfrak{a}}^{1}(M, L) \cong H_{\mathfrak{a}}^{1}(M, L)$. Now use induction.

Here we present another cohomology theory, Tate local cohomology. We shall use it as a tool to study Gorenstein local cohomology modules.

Definition 5.7. Let $N$ be an $R$-module with $\operatorname{Gid}_{R} N<\infty$. Consider the complete coresolution $N \rightarrow \mathbf{E} \rightarrow \mathbf{T}$ of $N$. For any $i \in \mathbb{Z}$, we define the $i$ th Tate local cohomology module of $M$ and $N$ with respect to $\mathfrak{a}$ by the formula

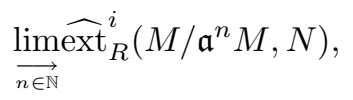

where $M$ is an arbitrary $R$-module. These modules will be denoted $\widehat{H}_{\mathfrak{a}}^{i}(M, N)$.

It is not difficult to see that these modules can be computed as follows: apply the left exact functor $\operatorname{Hom}_{R}\left(M, \Gamma_{\mathfrak{a}}()\right)$ to the complex $\mathbf{T}$ and take its $i$ th cohomology module. The result will be $\widehat{H}_{\mathfrak{a}}^{i}(M, N)$.

Lemma 5.8. Let $M$ be a finitely generated $R$-module. Then for any $R$-module $N$ of finite injective dimension, $\widehat{H}_{\mathfrak{a}}^{i}(M, N)=0$ for all $i>0$.

Proof. The result follows from Theorem 3.9.

Lemma 5.9. Let $E$ be an injective $R$-module. Then for any finitely generated $R$-module $M, H_{\mathfrak{a}}^{i}\left(\operatorname{Hom}_{R}(M, E)\right)=0$, for all $i>0$.

Proof. First note that, since local cohomology functor commutes with direct sums, we may reduce the lemma to the case $E=E(R / \mathfrak{p})$, for some prime ideal $\mathfrak{p}$. Using flat base change theorem [BS, 4.3.2], without loss of generality, we may assume that $R$ is local. But in this case $\operatorname{Hom}_{R}(M, E)$ is Artinian, and so by an appeal to the Grothendieck Vanishing Theorem we conclude the proof.

Theorem 5.10. Let $N$ be an R-module of finite $G$-injective dimension. Let $0 \rightarrow$ $M \rightarrow M^{\prime} \rightarrow M^{\prime \prime} \rightarrow 0$ be a short exact sequence of $R$-modules. Then there exists a long exact sequence of Tate local cohomology groups

$$
\cdots \rightarrow \widehat{H}_{\mathfrak{a}}^{i}\left(M^{\prime \prime}, N\right) \rightarrow \widehat{H}_{\mathfrak{a}}^{i}\left(M^{\prime}, N\right) \rightarrow \widehat{H}_{\mathfrak{a}}^{i}(M, N) \rightarrow \widehat{H}_{\mathfrak{a}}^{i+1}\left(M^{\prime \prime}, N\right) \rightarrow \cdots
$$

Proof. Consider a complete coresolution $N \rightarrow \mathbf{E} \rightarrow \mathbf{T}$ of $N$. For any integer $i$, the $i$ th term of $\mathbf{T}, T_{i}$ is injective. So the functor $\operatorname{Hom}_{R}\left(, T_{i}\right)$ is exact. Hence we obtain an exact sequence of complexes

$$
0 \rightarrow \operatorname{Hom}_{R}\left(M^{\prime \prime}, \mathbf{T}\right) \rightarrow \operatorname{Hom}_{R}\left(M^{\prime}, \mathbf{T}\right) \rightarrow \operatorname{Hom}_{R}(M, \mathbf{T}) \rightarrow 0
$$

By the previous lemma, applying $\Gamma_{\mathfrak{a}}$ on this exact sequence again gives a short exact sequence of complexes which in turn induces a long exact sequence of cohomology groups as desired. 
The following theorem provides a tight connection between Gorenstein, Tate and generalized local cohomology modules.

Theorem 5.11. Let $N$ be an $R$-module with $\operatorname{Gid}_{R} N=d$. Then for any $R$ module $M$, there exist natural in $M$ and $N$ homomorphisms $\bar{\gamma}_{R}^{n}(M, N)$, such that the following sequence is exact:

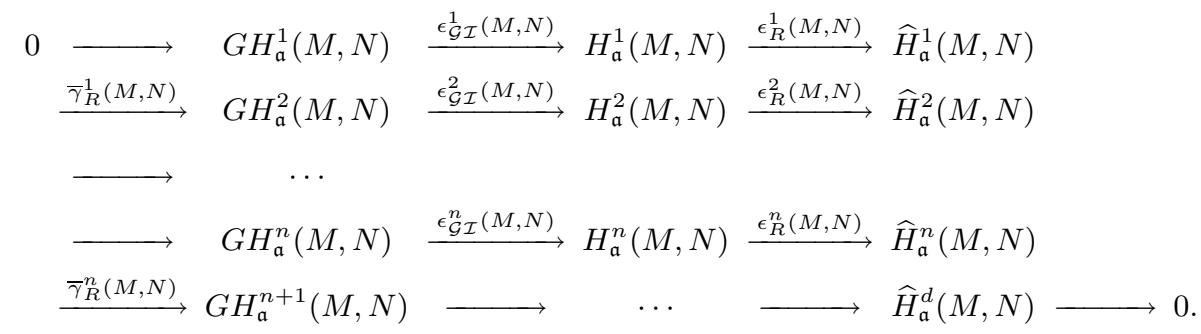

Proof. For any $n \in \mathbb{N}$, write the exact sequence of Theorem 3.10 for modules $M / \mathfrak{a}^{n} M$ and $N$. Then pass to the direct limit.

Corollary 5.12. If $\operatorname{pd}_{R} M<\infty$ and $N \in \widetilde{\mathcal{G I}}$ then $\widehat{H}_{\mathfrak{a}}^{i}(M, N)=0$ for all $i>0$. In particular, for $i>\operatorname{Gid}_{R} N$,

$$
G H_{\mathfrak{a}}^{i}(M, N)=H_{\mathfrak{a}}^{i}(M, N)=\widehat{H}_{\mathfrak{a}}^{i}(M, N)=0 .
$$

Proof. Use Proposition 5.6 in conjunction with the above theorem.

\section{VANishing ReSUlts OVER GORENSTEIN RINGS}

In this section we assume that $R$ is a commutative Noetherian Gorenstein ring. Our aim here is to provide some results concerning the vanishing (and non-vanishing) of Gorenstein local cohomology modules.

Proposition 6.1. Let $R$ be a Gorenstein ring of finite dimension and let $\mathfrak{a}$ be an ideal of $R$. Let $M$ be finitely generated and let $N$ be an $\mathfrak{a}$-torsion $R$-module. Then for all $i>0, G H_{\mathfrak{a}}^{i}(M, N) \cong \operatorname{ext}_{\mathcal{G I}}^{i}(M, N)$. In particular, for all $i>\mathrm{G}-\operatorname{dim}_{R} M$, $G H_{\mathfrak{a}}^{i}(M, N)=0$.

Proof. Since $R$ is Gorenstein of finite dimension, it is easy to see, using EJ2, 10.1.13], that the effect of $\Gamma_{\mathfrak{a}}$ on a G-injective $R$-module again gives a G-injective $R$-module. Using this we can construct a $\mathcal{G I}$-proper coresolution of $N$ in which each term is an $\mathfrak{a}$-torsion $R$-module. So for any $i \in \mathbb{Z}$, we have an isomorphism $G H_{\mathfrak{a}}^{i}(M, N) \cong \operatorname{ext}_{\mathcal{G I}}^{i}(M, N)$. By Theorem 4.3, the latter module is isomorphic to $\operatorname{Ext}_{\mathcal{G}}^{i}(M, N)$. So the result follows from [AM, 4.2].

Theorem 6.2. Let $R$ be a Gorenstein ring of finite dimension. Let $\mathfrak{a}$ be an ideal of $R$ and let $M$ and $N$ be finitely generated $R$-modules. Then for all $i>\mathrm{G}-\operatorname{dim}_{R} M+$ $\operatorname{dim} N, G H_{\mathfrak{a}}^{i}(M, N)=0$.

Proof. Consider the maps $\epsilon_{R}^{i}(M, N): H_{\mathfrak{a}}^{i}(M, N) \longrightarrow \widehat{H}_{\mathfrak{a}}^{i}(M, N)$ of Theorem 5.11. It is enough for us to show that for $i=\mathrm{G}-\operatorname{dim}_{R} M+\operatorname{dim} N, \epsilon_{R}^{i}(M, N)$ is an epimorphism and for $i>\mathrm{G}-\operatorname{dim}_{R} M+\operatorname{dim} N$ is an isomorphism. We do this by induction on $\operatorname{dim} N$. So suppose $\operatorname{dim} N=0$. In this case $N$ is a-torsion and so by the previous proposition for all $i>0, G H_{\mathfrak{a}}^{i}(M, N) \cong \operatorname{ext}_{\mathcal{G I}}^{i}(M, N)$. That is, for any $i>\mathrm{G}-\operatorname{dim}_{R} M, \epsilon^{i}$ s are an isomorphism and by Theorem 5.11, $\epsilon^{\mathrm{G}-\operatorname{dim}_{R} M}$ is an epimorphism. Now suppose inductively that $\operatorname{dim} N>0$. If $\Gamma_{\mathfrak{a}}(N)=N$, the result 
follows from the previous case. So suppose $\Gamma_{\mathfrak{a}}(N) \neq N$ and consider the short exact sequence

$$
0 \longrightarrow \Gamma_{\mathfrak{a}}(N) \longrightarrow N \longrightarrow N / \Gamma_{\mathfrak{a}}(N) \longrightarrow 0
$$

We obtain the commutative diagram

$$
\begin{aligned}
& H_{\mathfrak{a}}^{i}\left(M, \Gamma_{\mathfrak{a}}(N)\right) \longrightarrow H_{\mathfrak{a}}^{i}(M, N) \longrightarrow H_{\mathfrak{a}}^{i}\left(M, \frac{N}{\Gamma_{\mathfrak{a}}(N)}\right) \longrightarrow H_{\mathfrak{a}}^{i+1}\left(M, \Gamma_{\mathfrak{a}}(N)\right) \\
& \downarrow \epsilon_{R}^{i}\left(M, \Gamma_{\mathfrak{a}}(N)\right) \quad \downarrow \quad \downarrow \epsilon_{R}^{i}(M, N) \quad \downarrow \epsilon_{R}^{i}\left(M, \frac{N}{\Gamma_{\mathfrak{a}}(N)}\right) \quad \downarrow \epsilon_{R}^{i+1}\left(M, \Gamma_{\mathfrak{a}}(N)\right) \\
& \widehat{H}_{\mathfrak{a}}^{i}\left(M, \Gamma_{\mathfrak{a}}(N)\right) \longrightarrow \widehat{H}_{\mathfrak{a}}^{i}(M, N) \longrightarrow \widehat{H}_{\mathfrak{a}}^{i}\left(M, \frac{N}{\Gamma_{\mathfrak{a}}(N)}\right) \longrightarrow \widehat{H}_{\mathfrak{a}}^{i+1}\left(M, \Gamma_{\mathfrak{a}}(N)\right)
\end{aligned}
$$

By a simple diagram chasing argument we see that it is enough for us to prove the result for $N / \Gamma_{\mathfrak{a}}(N)$. So passing to $N / \Gamma_{\mathfrak{a}}(N)$, we may assume that $\mathfrak{a}$ contains a nonzerodivisor on $N$, say $x$. The short exact sequence $0 \longrightarrow N \stackrel{x}{\longrightarrow} N \longrightarrow N / x N \longrightarrow 0$ induces a commutative diagram

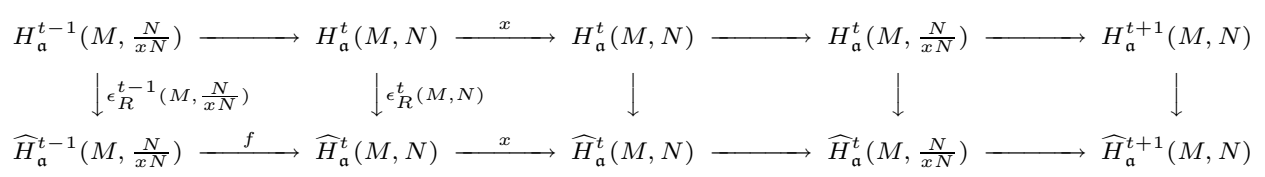

Consider the case $\mathrm{G}-\operatorname{dim}_{R} M+\operatorname{dim} N=t$. Then by the induction assumption $\epsilon_{R}^{t-1}(M, N / x N)$ is an epimorphism, and for all $i \geq t, \epsilon_{R}^{i}(M, N / x N)$ is an isomorphism. Let $\alpha \in \widehat{H}_{\mathfrak{a}}^{t}(M, N)$. There exists an integer $n$ such that $x^{n} \alpha=0$. Without loss of generality we may assume that $n=1$ and so $\alpha$ belongs to $\operatorname{Im} f$. So this concludes the proof that $\epsilon_{R}^{t}(M, N)$ is an epimorphism. The other isomorphisms follow easily by diagram chasing.

Corollary 6.3. Let $R$ be a (not necessarily finite-dimensional) Gorenstein ring. Let $M$ and let $N$ be finitely generated $R$-modules and let $N \in \overline{\mathcal{G I}}$. Then for any $i>\mathrm{G}_{-} \operatorname{dim}_{R} M+\operatorname{dim} N, G H_{\mathfrak{a}}^{i}(M, N)=0$.

Proof. Let $\mathfrak{p} \in \operatorname{Spec}(R)$ be an arbitrary prime. In view of Proposition 4.5,

$$
\left(G H_{\mathfrak{a}}^{i}(M, N)\right)_{\mathfrak{p}} \cong G H_{\mathfrak{a} R_{\mathfrak{p}}}^{i}\left(M_{\mathfrak{p}}, N_{\mathfrak{p}}\right) .
$$

By [AB], G- $\operatorname{dim}_{R_{\mathfrak{p}}} M_{\mathfrak{p}} \leq \mathrm{G}-\operatorname{dim}_{R} M$. We finish the argument by using the previous theorem.

Now let $(R, \mathfrak{m})$ be a commutative Noetherian Gorenstein local ring, let $M$ be a finitely generated $R$-module and let $N$ be an $R$-module. Since for any $n \in \mathbb{N}$, the $R$-module $M / \mathfrak{m}^{n} M$ is Artinian, it is G-perfect of G-dimension $d=\operatorname{dim} R$. So by [AM, 6.3], there exists an exact sequence as

$$
\begin{aligned}
& 0 \longrightarrow \widehat{\operatorname{Ext}}_{R}^{-1}\left(\frac{M}{\mathfrak{m}^{n} M}, N\right) \longrightarrow \operatorname{Tor}_{d}^{R}\left(\operatorname{Ext}_{R}^{d}\left(\frac{M}{\mathfrak{m}^{n} M}, R\right), N\right) \\
& \longrightarrow \operatorname{Ext}_{R}^{0}\left(\frac{M}{\mathfrak{m}^{n} M}, N\right) \longrightarrow \quad \ldots \quad \longrightarrow \quad \operatorname{Tor}_{d-r}^{R}\left(\operatorname{Ext}_{R}^{d}\left(\frac{M}{\mathfrak{m}^{n} M}, R\right), N\right) \\
& \longrightarrow \operatorname{Ext}_{R}^{r}\left(\frac{M}{\mathfrak{m}^{n} M}, N\right) \longrightarrow{\widehat{\operatorname{Ext}_{R}}}_{R}^{r}\left(\frac{M}{\mathfrak{m}^{n} M}, N\right) \longrightarrow \operatorname{Tor}_{d-r-1}^{R}\left(\operatorname{Ext}_{R}^{d}\left(\frac{M}{\mathfrak{m}^{n} M}, R\right), N\right) \\
& \cdots \longrightarrow \operatorname{Ext}_{R}^{d}\left(\frac{M}{\mathfrak{m}^{n} M}, N\right) \longrightarrow \widehat{\operatorname{Ext}}_{R}^{d}\left(\frac{M}{\mathfrak{m}^{n} M}, N\right) \longrightarrow 0
\end{aligned}
$$


By Theorem 4.8, $\widehat{\operatorname{Ext}}_{R}^{i} \cong \widehat{\operatorname{ext}}_{R}^{i}$ for all $i \in \mathbb{Z}$. So by taking the direct limit and by noting that 'Tor' commutes with direct limit, we get the following exact sequence:

$$
\begin{array}{ccccc}
0 & \stackrel{\epsilon_{R}^{-1}(M, N)}{\longrightarrow} \widehat{H}_{\mathfrak{m}}^{-1}(M, N) & \longrightarrow & \operatorname{Tor}_{d}^{R}\left(H_{\mathfrak{m}}^{d}(M, R), N\right) \\
\longrightarrow H_{\mathfrak{m}}^{0}(M, N) \stackrel{\epsilon_{R}^{0}(M, N)}{\longrightarrow} \mathrm{N}^{r} & \longrightarrow \operatorname{Tor}_{d-r}^{R}\left(H_{\mathfrak{m}}^{d}(M, R), N\right) \\
\cdots & \longrightarrow H_{\mathfrak{m}}^{r}(M, N) \stackrel{\epsilon_{R}^{r}(M, N)}{\longrightarrow} \widehat{H}_{\mathfrak{m}}^{r}(M, N) & \longrightarrow \operatorname{Tor}_{d-r-1}^{R}\left(H_{\mathfrak{m}}^{d}(M, R), N\right) \\
\cdots & H_{\mathfrak{m}}^{d}(M, N) \stackrel{\epsilon_{R}^{d}(M, N)}{\longrightarrow} \widehat{H}_{\mathfrak{m}}^{d}(M, N) & \longrightarrow & 0
\end{array}
$$

But since $R$ is Gorenstein, one can use its standard minimal injective coresolution to deduce that $H_{\mathfrak{m}}^{d}(M, R) \cong \operatorname{Hom}_{R}(M, E(k))$, and so the standard isomorphism in homology implies the isomorphisms

$$
\begin{aligned}
\operatorname{Tor}_{i}^{R}\left(H_{\mathfrak{m}}^{d}(M, R), N\right) & \cong \operatorname{Tor}_{i}^{R}\left(\operatorname{Hom}_{R}(M, E(k)), N\right) \\
& \cong \operatorname{Hom}_{R}\left(\operatorname{Ext}_{R}^{i}(N, M), E(k)\right) .
\end{aligned}
$$

So if $D()=\operatorname{Hom}_{R}(, E(k))$ is the Matlis duality functor, we can rewrite the above exact sequence as follows:

$$
\begin{aligned}
& 0 \quad \stackrel{\epsilon_{R}^{-1}(M, N)}{\longrightarrow} \widehat{H}_{\mathfrak{m}}^{-1}(M, N) \longrightarrow \quad D\left(\operatorname{Ext}_{R}^{d}(N, M)\right) \\
& \longrightarrow H_{\mathfrak{m}}^{0}(M, N) \stackrel{\epsilon_{R}^{0}(M, N)}{\longrightarrow} \quad \cdots \quad \longrightarrow D\left(\operatorname{Ext}_{R}^{d-r}(N, M)\right) \\
& \longrightarrow H_{\mathfrak{m}}^{r}(M, N) \stackrel{\epsilon_{R}^{r}(M, N)}{\longrightarrow} \widehat{H}_{\mathfrak{m}}^{r}(M, N) \longrightarrow D\left(\operatorname{Ext}_{R}^{d-r-1}(N, M)\right) \\
& \cdots \longrightarrow H_{\mathfrak{m}}^{d}(M, N) \stackrel{\epsilon_{R}^{d}(M, N)}{\longrightarrow} \widehat{H}_{\mathfrak{m}}^{d}(M, N) \longrightarrow 0
\end{aligned}
$$

Hence we have the following proposition.

Proposition 6.4. Let $(R, \mathfrak{m})$ be a Gorenstein local ring. Fix $n \in \mathbb{N}$. With the above notation, the following conditions are equivalent:

i) $\epsilon_{R}^{i}(M, N)$ is an isomorphism for all $i<n$ and $\epsilon_{R}^{n}(M, N)$ is a monomorphism.

ii) $\operatorname{Ext}_{R}^{d-i}(N, M)=0$ for all $i \leq n$.

As a corollary we have a vanishing result for Gorenstein local cohomology modules.

Corollary 6.5. Let $(R, \mathfrak{m})$ be a Gorenstein local ring. For $n \in \mathbb{N}$ if we have one (and hence both) of the equivalent conditions in the above lemma, then $G H_{\mathfrak{m}}^{i}(M, N)$ $=0$ for all $i \leq n$.

Proof. Let $\operatorname{Ext}_{R}^{d-i}(N, M)=0$ for all $i \leq n$. So by the above isomorphisms, $\operatorname{Tor}_{i}^{R}\left(H_{\mathfrak{m}}^{d}(M, R), N\right)=0$. This in conjunction with [AM, 6.3(3)] implies the vanishing of $\underset{n \in \mathbb{N}}{\lim } \operatorname{Ext}_{\mathcal{G}}^{i}\left(M / \mathfrak{m}^{n} M, N\right)$ for all $i \leq n$. But since $R$ is Gorenstein,

$$
\operatorname{Ext}_{\mathcal{G}}^{i}\left(M / \mathfrak{m}^{n} M, N\right) \cong \operatorname{ext}_{\mathcal{G} \mathcal{I}}^{i}\left(M / \mathfrak{m}^{n} M, N\right)
$$

for all $i \in \mathbb{Z}$ and all $n \in \mathbb{N}$. The result now follows.

The following corollary should be compared with the Grothendieck local duality for ordinary local cohomology module. 
Corollary 6.6. Suppose that $(R, \mathfrak{m})$ is a Gorenstein local ring of dimension d. Set $D()=\operatorname{Hom}_{R}(, E(k))$. Then for any $2 \leq i \leq d$, there is an isomorphism

$$
G H_{\mathfrak{m}}^{i}(M, N) \cong D\left(\operatorname{Ext}_{R}^{d-i}(N, M)\right) \text {. }
$$

Moreover for $i=1$, there is an epimorphism

$$
D\left(\operatorname{Ext}_{R}^{d-1}(N, M)\right) \longrightarrow G H_{\mathfrak{m}}^{1}(M, N)
$$

Proof. Compare the long exact sequence of Theorem 5.11 with the above exact sequence. For the epimorphism, see [AM, 6.3].

\section{ACKNOWLEDGMENTS}

We thank Professor Edgar Enochs for reading the first draft of the manuscript. He informed us that Alina Iacob had found an independent proof of the fact that one can compute the Tate cohomology using G-injective coresolutions. The authors also

thank the referee for many considerable comments and suggestions that improved the paper.

\section{REFERENCES}

[AB] M. Auslander, M. Bridger, Stable module theory, Mem. Amer. Math. Soc. 94 (1969). MR.0269685 (42:4580)

[ADS] M. Auslander, S. Ding, and $\varnothing$. Solberg, Liftings and weak liftings of modules, J. Algebra 156 (1969) 273-317. MR1216471 (94d:16007)

[AM] L.L. Avramov, A. Martsinkovsky, Absolute, relative, and Tate cohomology of modules of finite Gorenstein dimension, Proc. London Math. Soc. (3) 85 (2002), no. 2, 393-440. MR.1912056 (2003g:16009)

[B] M. H. Bijan-Zadeh, A common generalization of local cohomology theories, Glasgow Math. J. 21 (1980), no. 2, 173-181. MR0582127 (82e:13012)

[BS] M. Brodmann, R.Y. Sharp, 'Local cohomology - an algebraic introduction with geometric applications', Cambridge studies in advanced mathematics No. 60, Cambridge University Press (1998). MR1613627 (99h:13020)

[Bu] R.-O. Buchweitz, Maximal Cohen-Macaulay modules and Tate cohomology over Gorenstein rings, Preprint, Univ. Hannover, 1986.

[CK] J. Cornick, P.H. Kropholler, On complete resolutions, Topology Appl. 78 (1997), 235-250. MR.1454602 (98k:20087)

[EM] S. Eilenberg, J.C. Moore, Fundations of relative homological algebra, Mem. Amer. Math. Soc. Comm. Algebra 55, 1965. MR0178036 (31:2294)

[EJ1] E. E. Enochs, O. M.G. Jenda, Resolutions by Gorenstein injective and projective modules and modules of finite injective dimension over Gorenstein rings, Comm. Algebra 23 (1995), no. 3, 869-877. MR1316737 (96m:16010)

[EJ2] E. E. Enochs, O. M.G. Jenda, Relative homological algebra, de Gruyter Expositions in Mathematics 30, 2000. MR 1753146 (2001h:16013)

[G] S. Goto, Vanishing of $\operatorname{Ext}_{A}^{i}(M, A)$, J. Math. Kyoto Univ. 22 (1982), 481-484. MR0674605 (84c:13019)

[Gr] A. Grothendieck, 'Cohomologie Locale des Faisceaux et Theoremes de Lefschetz Locaux et Globaux', SGA 2, North Holland, 1969. MR0476737 (57:16294)

[He] J. Herzog, Komplexe, Aufösungen und dualität in der lokalen Algebra, Preprint, Universität Regensburg, 1974.

[H1] H. Holm, Gorenstein derived functors, Proc. Amer. Math. Soc. 132 (2004), 1913-1923. MR2053961

[H2] H. Holm, Gorenstein Homological dimensions, J. Pure Appl. Algebra 189 (2004), 167-193. MR2038564 (2004k:16013)

[M] A. Martsinkovsky, New homological invariants for modules over local rings, I, J. Pure Appl. Algebra 110 (1996), 1-8. MR1390668 (97d:13019)

[Ma] S. Mac Lane, Homology, Grundlehren Math. Wiss. 114, Springer, Berlin, 1967. MR0349792 $(50: 2285)$ 
[N] B. E. A. Nucinkis, Complete cohomology for arbitrary rings using injectives, J. Pure Appl. Algebra 131 (1998), no. 3, 297-318. MR1637023 (99h:16012)

[R] J. Rotman, An introduction to homological algebra, Academic Press, New York, 1979. MR0538169 (80k:18001)

School of Mathematics, Institute for Studies in Theoretical Physics and MathematiCs (IPM), P.O. Box 19395-5746, Tehran, Iran - AND - Shahre-Kord University, P.O. Box 115, Shahre-Kord, Iran

E-mail address: Asadollahi@ipm.ir

School of Mathematics, Institute for Studies in Theoretical Physics and Mathematics (IPM), P.O. Box 19395-5746, Tehran, Iran - and - Department of Mathematics, University of Isfahan, P.O. Box 81746-73441, Isfahan, Iran

E-mail address: Salarian@ipm.ir 